\title{
Robust Filtering of Sequences with Periodically Stationary Multiplicative Seasonal Increments
}

\author{
Maksym Luz ${ }^{1}$, Mikhail Moklyachuk ${ }^{2, *}$ \\ ${ }^{1}$ BNP Paribas Cardif, Kyiv, Ukraine \\ ${ }^{2}$ Taras Shevchenko National University of Kyiv, Ukraine
}

\begin{abstract}
We consider stochastic sequences with periodically stationary generalized multiple increments of fractional order which combines cyclostationary, multi-seasonal, integrated and fractionally integrated patterns. We solve the filtering problem for linear functionals constructed from unobserved values of a stochastic sequence of this type based on observations of the sequence with a periodically stationary noise sequence. For sequences with known matrices of spectral densities, we obtain formulas for calculating values of the mean square errors and the spectral characteristics of the optimal filtering of the functionals. Formulas that determine the least favourable spectral densities and the minimax (robust) spectral characteristics of the optimal linear filtering of the functionals are proposed in the case where spectral densities of the sequences are not exactly known while some sets of admissible spectral densities are given.
\end{abstract}

Keywords Periodically Stationary Sequence, SARFIMA, Fractional Integration, Filtering, Optimal Linear Estimate, Mean Square Error, Least Favourable Spectral Density Matrix, Minimax Spectral Characteristics.

AMS 2010 subject classifications 60G10, 60G25, 60G35, 62M20, 62P20,93E10, 93E11

DOI:10.19139/soic-2310-5070-1197

\section{Introduction}

Non-stationary and long memory time series models are wildly used in different fields of economics, finance, climatology, air pollution, signal processing etc. (see, for example, papers by Dudek and Hurd [9], Johansen and Nielsen [22], Reisen et al.[44]). A core example - a general multiplicative model, or $S A R I M A(p, d, q) \times$ $(P, D, Q)_{s}$ - was introduced in the book by Box and Jenkins et al. [5]. It includes both integrated and seasonal factors:

$$
\Psi\left(B^{s}\right) \psi(B)(1-B)^{d}\left(1-B^{s}\right)^{D} x_{t}=\Theta\left(B^{s}\right) \theta(B) \varepsilon_{t},
$$

where $\left\{\varepsilon_{t}\right\}$ is a sequence of i.i.d. random variables, and where $\Psi(z)$ and $\Theta(z)$ are two polynomials of degrees of $P$ and $Q$ respectively which have roots outside the unit circle. The parameters $d$ and $D$ are allowed to be fractional. When $|d+D|<1 / 2$ and $|D|<1 / 2$, the process (1) is stationary and invertible. The paper by Porter-Hudak [43] illustrates an application of a seasonal ARFIMA model to the analysis of the monetary aggregates used by U.S. Federal Reserve. Another model of fractional integration is GARMA processes described by the equation (see Gray, Cheng and Woodward [16])

$$
\left(1-2 u B+B^{2}\right)^{d} x_{t}=\varepsilon_{t}, \quad|u| \leq 1 .
$$

For the resent results dedicated to the statistical inference for seasonal long-memory sequences, we refer to the paper by Tsai, Rachinger and Lin [48], who developed methods of estimation of parameters in case of measurement

\footnotetext{
*Correspondence to: Mikhail Moklyachuk (Email: Moklyachuk@gmail.com). Department of Probability Theory, Statistics and Actuarial Mathematics, Taras Shevchenko National University of Kyiv, Volodymyrska 64 Str., Kyiv 01601, Ukraine.
}

ISSN 2310-5070 (online) ISSN 2311-004X (print)

Copyright $(0) 2021$ International Academic Press 
errors. In their paper Baillie, Kongcharoen and Kapetanios [3] compared MLE and semiparametric estimation procedures for prediction problems based on ARFIMA models. Based on simulation study, they indicate better performance of MLE predictor than the one based on the two-step local Whittle estimation. Hassler and Pohle [19] (see also Hassler [18]) assess a predictive performance of various methods of forecasting of inflation and return volatility time series and show strong evidences for models with a fractional integration component.

Another type of non-stationary processes are periodically correlated, or cyclostationary, processes introduced by Gladyshev [13], which belong to the class of processes with time-dependent spectrum and are widely used in signal processing and communications (see Napolitano [40] for a review of recent works on cyclostationarity and its applications). Periodic time series are also considered as extension of seasonal models [2, 4, 29, 41].

One of the fields of interests related to time series analysis is optimal filtering. It aims to remove the unobserved components, such as trends, seasonality or noise signal, from the observed data $[1,6]$.

Methods of parameters estimations and filtering usually do not take into account the issues arising from real data, namely, the presence of outliers, measurement errors, incomplete information about the spectral, or model, structure etc. From this point of view, we see an increasing interest to robust methods of estimation that are reasonable in such cases (see Reisen, et al. [45], Solci at al. [47] for the examples of robust estimates of SARIMA and PAR models). Grenander [15], Hosoya [20], Kassam [24], Franke [10], Vastola and Poor [49], Moklyachuk [35, 36], Luz and Moklyachuk [31], Liu et al. [28] studied minimax extrapolation, interpolation and filtering problems for stationary sequences and processes.

This article is dedicated to the robust filtering of stochastic sequences with periodically stationary long memory multiple seasonal increments, or sequences with periodically stationary general multiplicative (GM) increments, introduced by Luz and Moklyachuk [33]. In the recent years, models with multiple seasonal and periodic patterns are in scope of interest, see Dudek [8], Gould et al. [14] and Reisen et al. [44], Hurd and Piparas [21]. This motivates us to study the time series combining a periodic structure of the covariation function as well as multiple seasonal factors. The discussed problem is a natural continuation of the researches on minimax filtering of stationary vector-valued processes, periodically correlated processes and processes with stationary increments which have been performed by Moklyachuk and Masyutka [37], Moklyachuk and Golichenko (Dubovetska) [7], Luz and Moklyachuk [30, 32] respectively. We also mention the works by Moklyachuk, Masyutka and Sidei [38, 39, 34], who derive minimax estimates of stationary processes from observations with missed values, and the work by Kozak and Moklyachuk [27], where the interpolation problem for stochastic sequences with periodically stationary increments was studied.

The article is organized as follows. In Section 2, we recall definitions of generalized multiple (GM) increment sequence $\chi_{\bar{\mu}, \bar{s}}^{(d)}(\vec{\xi}(m))$ and stochastic sequences $\xi(m)$ with periodically stationary (periodically correlated, cyclostationary) GM increments. The spectral theory of vector-valued GM increment sequences is discussed. Section 3 deals with the classical filtering problem for linear functionals $A \xi$ and $A_{N} \xi$ which are constructed from unobserved values of the sequence $\xi(m)$ when the spectral densities of the sequence $\xi(m)$ and a noise sequence $\eta(m)$ are known. Estimates are obtained by applying the Hilbert space projection technique to the vector sequence $\vec{\xi}(m)+\vec{\eta}(m)$ with stationary GM increments under the stationary noise sequence $\vec{\eta}(m)$ uncorrelated with $\vec{\xi}(m)$. The case of non-stationary fractional integration is discussed as well. Section 4 is dedicated to the minimax (robust) estimates in cases, where spectral densities of sequences are not exactly known while some sets of admissible spectral densities are specified. We illustrate the proposed technique in the case of particular types of the sets, which are generalizations of the sets of admissible spectral densities described in a survey article by Kassam and Poor [25] for stationary stochastic processes..

\section{Stochastic sequences with periodically stationary generalized multiple increments}

\subsection{Preliminary notations and definitions}

Consider a stochastic sequence $\xi(m), m \in \mathbb{Z}$, and a backward shift operator $B_{\mu}$ with the step $\mu \in \mathbb{Z}$, such that $B_{\mu} \xi(m)=\xi(m-\mu) ; B:=B_{1}$. Then $B_{\mu}^{s}=B_{\mu} B_{\mu} \cdot \ldots \cdot B_{\mu}$. Define an incremental operator $\chi_{\bar{\mu}, \bar{s}}^{(d)}(B)=$ 
$\left(1-B_{\mu_{1}}^{s_{1}}\right)^{d_{1}}\left(1-B_{\mu_{2}}^{s_{2}}\right)^{d_{2}} \cdot \ldots \cdot\left(1-B_{\mu_{r}}^{s_{r}}\right)^{d_{r}}$, where $d:=d_{1}+d_{2}+\ldots+d_{r}, \bar{d}=\left(d_{1}, d_{2}, \ldots, d_{r}\right) \in\left(\mathbb{N}^{*}\right)^{r}, \bar{s}=$ $\left(s_{1}, s_{2}, \ldots, s_{r}\right) \in\left(\mathbb{N}^{*}\right)^{r}$ and $\bar{\mu}=\left(\mu_{1}, \mu_{2}, \ldots, \mu_{r}\right) \in\left(\mathbb{N}^{*}\right)^{r}$ or $\in(\mathbb{Z} \backslash \mathbb{N})^{r}$. Here $\mathbb{N}^{*}=\mathbb{N} \backslash\{0\}$.

Within the article, $\delta_{l p}$ denotes Kronecker symbols, $\left(\begin{array}{l}n \\ l\end{array}\right)=\frac{n !}{l !(n-l) !}$.

\section{Definition 1}

For a stochastic sequence $\xi(m), m \in \mathbb{Z}$, the sequence

$$
\begin{aligned}
\chi_{\bar{\mu}, \bar{s}}^{(d)}(\xi(m)) & :=\chi_{\bar{\mu}, \bar{s}}^{(d)}(B) \xi(m)=\left(1-B_{\mu_{1}}^{s_{1}}\right)^{d_{1}}\left(1-B_{\mu_{2}}^{s_{2}}\right)^{d_{2}} \cdot \ldots \cdot\left(1-B_{\mu_{r}}^{s_{r}}\right)^{d_{r}} \xi(m) \\
& =\sum_{l_{1}=0}^{d_{1}} \ldots \sum_{l_{r}=0}^{d_{r}}(-1)^{l_{1}+\ldots+l_{r}}\left(\begin{array}{c}
d_{1} \\
l_{1}
\end{array}\right) \cdot \ldots \cdot\left(\begin{array}{c}
d_{r} \\
l_{r}
\end{array}\right) \xi\left(m-\mu_{1} s_{1} l_{1}-\cdots-\mu_{r} s_{r} l_{r}\right)
\end{aligned}
$$

is called a stochastic generalized multiple (GM) increment sequence of differentiation order $d$ with a fixed seasonal vector $\bar{s} \in\left(\mathbb{N}^{*}\right)^{r}$ and a varying step $\bar{\mu} \in\left(\mathbb{N}^{*}\right)^{r}$ or $\in(\mathbb{Z} \backslash \mathbb{N})^{r}$.

The multiplicative increment operator $\chi_{\bar{\mu}, \bar{s}}^{(d)}(B)$ admits a representation

$$
\chi_{\bar{\mu}, \bar{s}}^{(d)}(B)=\prod_{i=1}^{r}\left(1-B_{\mu_{i}}^{s_{i}}\right)^{d_{i}}=\sum_{k=0}^{n(\gamma)} e_{\gamma}(k) B^{k},
$$

where $n(\gamma):=\sum_{i=1}^{r} \mu_{i} s_{i} d_{i}$. The explicit representation of the coefficients $e_{\gamma}(k)$ is given in [33].

Definition 2

A stochastic GM increment sequence $\chi_{\bar{\mu}, \bar{s}}^{(d)}(\xi(m))$ is called a wide sense stationary if the mathematical expectations

$$
\begin{aligned}
\mathrm{E} \chi_{\bar{\mu}, \bar{s}}^{(d)}\left(\xi\left(m_{0}\right)\right) & =c_{\bar{s}}^{(d)}(\bar{\mu}), \\
\mathrm{E} \chi_{\bar{\mu}_{1}, \bar{s}}^{(d)}\left(\xi\left(m_{0}+m\right)\right) \chi_{\bar{\mu}_{2}, \bar{s}}^{(d)}\left(\xi\left(m_{0}\right)\right) & =D_{\bar{s}}^{(d)}\left(m ; \bar{\mu}_{1}, \bar{\mu}_{2}\right)
\end{aligned}
$$

exist for all $m_{0}, m, \bar{\mu}, \bar{\mu}_{1}, \bar{\mu}_{2}$ and do not depend on $m_{0}$. The function $c_{\bar{s}}^{(d)}(\bar{\mu})$ is called a mean value and the function $D_{\bar{s}}^{(d)}\left(m ; \bar{\mu}_{1}, \bar{\mu}_{2}\right)$ is called a structural function of the stationary GM increment sequence (of a stochastic sequence with stationary GM increments).

The stochastic sequence $\xi(m), m \in \mathbb{Z}$ determining the stationary GM increment sequence $\chi_{\bar{\mu}, \bar{s}}^{(d)}(\xi(m))$ by (3) is called a stochastic sequence with stationary GM increments (or GM increment sequence of order $d$ ).

\section{Remark 1}

Spectral properties of one-pattern increment sequence $\chi_{\mu, 1}^{(n)}(\xi(m)):=\xi^{(n)}(m, \mu)=\left(1-B_{\mu}\right)^{n} \xi(m)$ and the continuous time increment process $\xi^{(n)}(t, \tau)=\left(1-B_{\tau}\right)^{n} \xi(t)$ are described in [50], [51].

\subsection{Definition and spectral representation of stochastic sequences with periodically stationary GM increment}

In this section, we present definition, justification and a brief review of the spectral theory of stochastic sequences with periodically stationary multiple seasonal increments.

Definition 3

A stochastic sequence $\xi(m), m \in \mathbb{Z}$ is called a stochastic sequence with periodically stationary (periodically correlated) GM increments with period $T$ if the mathematical expectations

$$
\begin{aligned}
\mathrm{E} \chi_{\bar{\mu}, T \bar{s}}^{(d)}(\xi(m+T)) & =\mathrm{E} \chi_{\bar{\mu}, T \bar{s}}^{(d)}(\xi(m))=c_{T \bar{s}}^{(d)}(m, \bar{\mu}), \\
\mathrm{E} \chi_{\bar{\mu}_{1}, T \bar{s}}^{(d)}(\xi(m+T)) \chi_{\bar{\mu}_{2}, T \bar{s}}^{(d)}(\xi(k+T)) & =D_{T \bar{s}}^{(d)}\left(m+T, k+T ; \bar{\mu}_{1}, \bar{\mu}_{2}\right)=D_{T \bar{s}}^{(d)}\left(m, k ; \bar{\mu}_{1}, \bar{\mu}_{2}\right)
\end{aligned}
$$

exist for every $m, k, \bar{\mu}_{1}, \bar{\mu}_{2}$ and $T>0$ is the least integer for which these equalities hold. 
It follows from Definition 3 that the sequence

$$
\xi_{p}(m)=\xi(m T+p-1), \quad p=1,2, \ldots, T ; \quad m \in \mathbb{Z}
$$

forms a vector-valued sequence $\vec{\xi}(m)=\left\{\xi_{p}(m)\right\}_{p=1,2, \ldots, T}, m \in \mathbb{Z}$ with stationary GM increments as follows:

$$
\begin{aligned}
\chi_{\bar{\mu}, \bar{s}}^{(d)}\left(\xi_{p}(m)\right) & =\sum_{l_{1}=0}^{d_{1}} \ldots \sum_{l_{r}=0}^{d_{r}}(-1)^{l_{1}+\ldots+l_{r}}\left(\begin{array}{c}
d_{1} \\
l_{1}
\end{array}\right) \cdot \ldots \cdot\left(\begin{array}{c}
d_{r} \\
l_{r}
\end{array}\right) \xi_{p}\left(m-\mu_{1} s_{1} l_{1}-\ldots-\mu_{r} s_{r} l_{r}\right) \\
& =\sum_{l_{1}=0}^{d_{1}} \ldots \sum_{l_{r}=0}^{d_{r}}(-1)^{l_{1}+\ldots+l_{r}}\left(\begin{array}{c}
d_{1} \\
l_{1}
\end{array}\right) \ldots \cdot\left(\begin{array}{c}
d_{r} \\
l_{r}
\end{array}\right) \xi\left(\left(m-\mu_{1} s_{1} l_{1}-\ldots-\mu_{r} s_{r} l_{r}\right) T+p-1\right) \\
& =\chi_{\bar{\mu}, T \bar{s}}^{(d)}(\xi(m T+p-1)), \quad p=1,2, \ldots, T,
\end{aligned}
$$

where $\chi_{\bar{\mu}, \bar{s}}^{(d)}\left(\xi_{p}(m)\right)$ is the GM increment of the $p$-th component of the vector-valued sequence $\vec{\xi}(m)$.

\section{Example 1}

Define a $T$-periodic seasonal autoregressive integrated moving average model (PSARIMA) $\left\{X_{m}, m \in \mathbb{Z}\right\}$, with multiple seasonal patterns by relation

$$
\phi_{m}(B)\left(1-B^{T}\right)^{d} \prod_{i=1}^{r} \Phi_{i, m}(B)\left(1-B^{T s_{i}}\right)^{d_{i}} X_{m}=\theta_{m}(B) \prod_{i=1}^{r} \Theta_{i, m}(B) \varepsilon_{m},
$$

where $\left\{\varepsilon_{m}\right\}$ is a sequence of i.i.d. random variables, and where all polynomials $\phi_{m}(z), \theta_{m}(z), \Phi_{i, m}(z), \Theta_{i, m}(z)$ are $T$-periodic by parameter $m$ functions, $1<s_{1}<\ldots<s_{r}$. Define

$$
\begin{aligned}
& \Phi_{m}(z):=\phi_{m}(z) \prod_{i=1}^{r} \Phi_{i, m}(z)=\sum_{k=0}^{q_{1}} \Phi_{m}(k) z^{k}, \\
& \Theta_{m}(z):=\theta_{m}(z) \prod_{i=1}^{r} \Theta_{i, m}(z)=\sum_{k=0}^{q_{2}} \Theta_{m}(k) z^{k}
\end{aligned}
$$

and put $\Phi_{m}(k)=0$ for $k>q_{1}, \Theta_{m}(k)=0$ for $k>q_{2}$. Then the increment sequence

$$
Y_{m}=\left(1-B^{T}\right)^{d} \prod_{i=1}^{r}\left(1-B^{T s_{i}}\right)^{d_{i}} X_{m}
$$

is periodically stationary and allows a stationary vector representation

$$
\mathbf{Y}_{m}=(1-B)^{d} \prod_{i=1}^{r}\left(1-B^{s_{i}}\right)^{d_{i}} \mathbf{X}_{m}
$$

with

$$
\mathbf{Y}_{m}=\left(Y_{m T}, Y_{m T+1}, \ldots, Y_{m T+T-1}\right)^{\top}, \quad \mathbf{X}_{m}=\left(X_{m T}, X_{m T+1}, \ldots, X_{m T+T-1}\right)^{\top},
$$

$\boldsymbol{\varepsilon}_{m}=\left(\varepsilon_{m T}, \varepsilon_{m T+1}, \ldots, \varepsilon_{m T+T-1}\right)^{\top}$. We can write the relation

$$
\boldsymbol{\Pi} \mathbf{Y}_{m}+\sum_{l=1}^{q_{1}^{*}} \boldsymbol{\Pi}_{l} \mathbf{Y}_{m-l}=\boldsymbol{\Xi} \boldsymbol{\varepsilon}_{m}+\sum_{l=1}^{q_{2}^{*}} \boldsymbol{\Xi}_{l} \boldsymbol{\varepsilon}_{m-l}
$$

where $\boldsymbol{\Pi}(k, j)=\Phi_{k}(k-j), \boldsymbol{\Xi}(k, j)=\Theta_{k}(k-j)$ for $k \geq j, \boldsymbol{\Pi}(k, j)=0, \boldsymbol{\Xi}(k, j)=0$ otherwise. $\boldsymbol{\Pi}_{l}(k, j)=$ $\Phi_{k}(l T+k-j), \boldsymbol{\Xi}_{l}(k, j)=\Theta_{k}(l T+k-j)$ [9], provided $\operatorname{det}\left(\boldsymbol{\Pi}+\sum_{l=1}^{q_{1}^{*}} \boldsymbol{\Pi}_{l} z^{l}\right) \neq 0$ for $|z| \leq 1$ [17].

A GM increment sequence is defined as

$$
\chi_{\bar{\mu}, \bar{s}}^{(d)}\left(\mathbf{X}_{m}\right)=\left(1-B^{\mu_{0}}\right)^{d} \prod_{i=1}^{r}\left(1-B^{s_{i} \mu_{i}}\right)^{d_{i}} \mathbf{X}_{m}, m \in \mathbb{Z} .
$$


The following theorem describes the spectral structure of the GM increment [23], [33].

\section{Theorem 1}

1. The mean value and the structural function of the vector-valued stochastic stationary GM increment sequence $\chi_{\bar{\mu}, \bar{s}}^{(d)}(\vec{\xi}(m))$ can be represented in the form

$$
\begin{aligned}
c_{\bar{s}}^{(d)}(\bar{\mu}) & =c \prod_{i=1}^{r} \mu_{i}^{d_{i}} \\
D_{\bar{s}}^{(d)}\left(m ; \bar{\mu}_{1}, \bar{\mu}_{2}\right) & =\int_{-\pi}^{\pi} e^{i \lambda m} \chi_{\bar{\mu}_{1}}^{(d)}\left(e^{-i \lambda}\right) \chi_{\bar{\mu}_{2}}^{(d)}\left(e^{i \lambda}\right) \frac{1}{\left|\beta^{(d)}(i \lambda)\right|^{2}} d F(\lambda),
\end{aligned}
$$

where

$$
\chi_{\bar{\mu}}^{(d)}\left(e^{-i \lambda}\right)=\prod_{j=1}^{r}\left(1-e^{-i \lambda \mu_{j} s_{j}}\right)^{d_{j}}, \quad \beta^{(d)}(i \lambda)=\prod_{j=1}^{r} \prod_{k_{j}=-\left[s_{j} / 2\right]}^{\left[s_{j} / 2\right]}\left(i \lambda-2 \pi i k_{j} / s_{j}\right)^{d_{j}},
$$

$c$ is a vector, $F(\lambda)$ is the matrix-valued spectral function of the stationary stochastic sequence $\chi_{\bar{\mu}, \bar{s}}^{(d)}(\vec{\xi}(m))$. The vector $c$ and the matrix-valued function $F(\lambda)$ are determined uniquely by the GM increment sequence $\chi_{\bar{\mu}, \bar{s}}^{(d)}(\vec{\xi}(m))$.

2. The stationary GM increment sequence $\chi_{\bar{\mu}, \bar{s}}^{(d)}(\vec{\xi}(m))$ admits the spectral representation

$$
\chi_{\bar{\mu}, \bar{s}}^{(d)}(\vec{\xi}(m))=\int_{-\pi}^{\pi} e^{i m \lambda} \chi_{\bar{\mu}}^{(d)}\left(e^{-i \lambda}\right) \frac{1}{\beta^{(d)}(i \lambda)} d \vec{Z}_{\xi^{(d)}}(\lambda),
$$

where $\vec{Z}_{\xi^{(d)}}(\lambda)=\left\{Z_{p}(\lambda)\right\}_{p=1}^{T}$ is a (vector-valued) stochastic process with uncorrelated increments on $[-\pi, \pi)$ connected with the spectral function $F(\lambda)$ by the relation

$$
\begin{gathered}
\mathrm{E}\left(Z_{p}\left(\lambda_{2}\right)-Z_{p}\left(\lambda_{1}\right)\right)\left(\overline{Z_{q}\left(\lambda_{2}\right)-Z_{q}\left(\lambda_{1}\right)}\right)=F_{p q}\left(\lambda_{2}\right)-F_{p q}\left(\lambda_{1}\right), \\
-\pi \leq \lambda_{1}<\lambda_{2}<\pi, \quad p, q=1,2, \ldots, T .
\end{gathered}
$$

Consider another vector-valued stochastic sequence with the stationary GM increments $\vec{\zeta}(m)=\vec{\xi}(m)+\vec{\eta}(m)$, where $\vec{\eta}(m)$ is a vector-valued stationary stochastic sequence, uncorrelated with $\vec{\xi}(m)$, with the spectral representation

$$
\vec{\eta}(m)=\int_{-\pi}^{\pi} e^{i \lambda m} d Z_{\eta}(\lambda)
$$

where $Z_{\eta}(\lambda)=\left\{Z_{\eta, p}(\lambda)\right\}_{p=1}^{T}, \lambda \in[-\pi, \pi)$, is a stochastic process with uncorrelated increments, that corresponds to the spectral function $G(\lambda)$ [17]. The stochastic stationary GM increment $\chi_{\bar{\mu}, \bar{s}}^{(d)}(\vec{\zeta}(m))$ allows the spectral representation

$$
\chi_{\bar{\mu}, \bar{s}}^{(d)}(\vec{\zeta}(m))=\int_{-\pi}^{\pi} e^{i \lambda m} \frac{\chi_{\bar{\mu}}^{(d)}\left(e^{-i \lambda}\right)}{\beta^{(d)}(i \lambda)} d Z_{\xi^{(n)}}(\lambda)+\int_{-\pi}^{\pi} e^{i \lambda m} \chi_{\bar{\mu}}^{(d)}\left(e^{-i \lambda}\right) d Z_{\eta}(\lambda),
$$

while $d Z_{\eta}(\lambda)=\left(\beta^{(d)}(i \lambda)\right)^{-1} d Z_{\eta^{(n)}}(\lambda), \lambda \in[-\pi, \pi)$. Therefore, in the case where the spectral functions $F(\lambda)$ and $G(\lambda)$ have the spectral densities $f(\lambda)$ and $g(\lambda)$, the spectral density $p(\lambda)=\left\{p_{i j}(\lambda)\right\}_{i, j=1}^{T}$ of the stochastic sequence $\vec{\zeta}(m)$ is determined by the formula

$$
p(\lambda)=f(\lambda)+\left|\beta^{(d)}(i \lambda)\right|^{2} g(\lambda) .
$$




\subsection{Stochastic sequences with GM fractional increments}

In the previous subsection, we describe the GM increment sequence $\chi_{\bar{\mu}, \bar{s}}^{(d)}(\vec{\xi}(m))$ of the positive integer orders $\left(d_{1}, \ldots, d_{r}\right)$. Here we consider the case of possibly fractional increment orders $d_{i}$.

Within the subsection, we put the step $\bar{\mu}=(1,1, \ldots, 1)$. Represent the increment operator $\chi_{\bar{s}}^{(d)}(B)$ in the form

$$
\chi_{\bar{s}}^{(R+D)}(B)=(1-B)^{R_{0}+D_{0}} \prod_{j=1}^{r}\left(1-B^{s_{j}}\right)^{R_{j}+D_{j}},
$$

where $(1-B)^{R_{0}+D_{0}}$ is an integrating component, $R_{j}, j=0,1, \ldots, r$, are non-negative integer numbers, $1<s_{1}<$ $\ldots<s_{r}$. Below we describe a representations $d_{j}=R_{j}+D_{j}, j=0,1, \ldots, r$, of the increment orders $d_{j}$ by stating conditions on the fractional parts $D_{j}$, such that the increment sequence $\vec{y}(m):=(1-B)^{R_{0}} \prod_{j=1}^{r}\left(1-B^{s_{j}}\right)^{R_{i}} \vec{\xi}(m)$ is a stationary fractionally integrated seasonal stochastic sequence. For example, in case of single increment pattern $\left(1-B^{s^{*}}\right)^{R^{*}+D^{*}}$, this condition is $\left|D^{*}\right|<1 / 2$.

A sequence $\chi_{\bar{s}}^{(R+D)}(\vec{\xi}(m))$ is called a fractional multiple $(F M)$ increment sequence.

Consider a generating function of the Gegenbauer polynomial:

$$
\left(1-2 u B+B^{2}\right)^{-d}=\sum_{n=0}^{\infty} C_{n}^{(d)}(u) B^{n},
$$

where

$$
C_{n}^{(d)}(u)=\sum_{k=0}^{[n / 2]} \frac{(-1)^{k}(2 u)^{n-2 k} \Gamma(d-k+n)}{k !(n-2 k) ! \Gamma(d)} .
$$

The following lemma and theorem hold true [33].

\section{Lemma 1}

Define the sets $\mathcal{M}_{j}=\left\{\nu_{k_{j}}=2 \pi k_{j} / s_{j}: k_{j}=0,1, \ldots,\left[s_{j} / 2\right]\right\}, j=0,1, \ldots, r$, and the set $\mathcal{M}=\bigcup_{j=0}^{r} \mathcal{M}_{j}$. Then the increment operator $\chi_{\bar{s}}^{(D)}(B):=(1-B)^{D_{0}} \prod_{j=1}^{r}\left(1-B^{s_{j}}\right)^{D_{j}}$ admits a representation

$$
\begin{aligned}
\chi_{\bar{s}}^{(D)}(B) & =\prod_{\nu \in \mathcal{M}}\left(1-2 \cos \nu B+B^{2}\right)^{\widetilde{D}_{\nu}} \\
& =(1-B)^{D_{0}+D_{1}+\ldots+D_{r}}(1+B)^{D_{\pi}} \prod_{\nu \in \mathcal{M} \backslash\{0, \pi\}}\left(1-2 \cos \nu B+B^{2}\right)^{D_{\nu}} \\
& =\left(\sum_{m=0}^{\infty} G_{k^{*}}^{+}(m) B^{m}\right)^{-1}=\sum_{m=0}^{\infty} G_{k^{*}}^{-}(m) B^{m}
\end{aligned}
$$

where

$$
\begin{aligned}
G_{k^{*}}^{+}(m) & =\sum_{0 \leq n_{1}, \ldots, n_{k^{*}} \leq m, n_{1}+\ldots+n_{k^{*}}=m} \prod_{\nu \in \mathcal{M}} C_{n_{\nu}}^{\left(\widetilde{D}_{\nu}\right)}(\cos \nu), \\
G_{k^{*}}^{-}(m) & \sum_{0 \leq n_{1}, \ldots, n_{k^{*}} \leq m, n_{1}+\ldots+n_{k^{*}}=m} \prod_{\nu \in \mathcal{M}} C_{n_{\nu}}^{\left(-\widetilde{D}_{\nu}\right)}(\cos \nu) .
\end{aligned}
$$

$k^{*}=|\mathcal{M}|, D_{\nu}=\sum_{j=0}^{r} D_{j} \mathbb{I}\left\{\nu \in \mathcal{M}_{j}\right\}, \widetilde{D}_{\nu}=D_{\nu}$ for $\nu \in \mathcal{M} \backslash\{0, \pi\}, \widetilde{D}_{\nu}=D_{\nu} / 2$ for $\nu=0$ and $\nu=\pi$.

Theorem 2

Assume that for a stochastic vector sequence $\vec{\xi}(m)$ and fractional differencing orders $d_{j}=R_{j}+D_{j}, j=$ $0,1, \ldots, r$, the FM increment sequence $\chi_{\overline{1}, \bar{s}}^{(R+D)}(\vec{\xi}(m))$ generated by increment operator (8) is a stationary sequence 
with a bounded from zero and infinity spectral density $\widetilde{f}_{\overline{1}}(\lambda)$. Then for the non-negative integer numbers $R_{j}$, $j=0,1, \ldots, r$, the GM increment sequence $\chi_{\overline{1}, \bar{s}}^{(R)}(\vec{\xi}(m))$ is stationary if $-1 / 2<D_{\nu}<1 / 2$ for all $\nu \in \mathcal{M}$, where $D_{\nu}$ are defined by real numbers $D_{j}, j=0,1, \ldots, r$, in Lemma 1 , and it is long memory if $0<D_{\nu}<1 / 2$ for at least one $\nu \in \mathcal{M}$, and invertible if $-1 / 2<D_{\nu}<0$. The spectral density $f(\lambda)$ of the stationary GM increment sequence $\chi_{\overline{1}, \bar{s}}^{(R)}(\vec{\xi}(m))$ admits a representation

$$
f(\lambda)=\left|\beta^{(R)}(i \lambda)\right|^{2}\left|\chi_{\overline{1}}^{(R)}\left(e^{-i \lambda}\right)\right|^{-2}\left|\chi_{\overline{1}}^{(D)}\left(e^{-i \lambda}\right)\right|^{-2} \widetilde{f}_{\overline{1}}(\lambda)=:\left|\chi_{\overline{1}}^{(D)}\left(e^{-i \lambda}\right)\right|^{-2} \tilde{f}(\lambda),
$$

where

$$
\begin{aligned}
\left|\chi_{\overline{1}}^{(D)}\left(e^{-i \lambda}\right)\right|^{-2} & =\left|\sum_{m=0}^{\infty} G_{k^{*}}^{+}(m) e^{-i \lambda m}\right|^{2}=\left|\sum_{m=0}^{\infty} G_{k^{*}}^{-}(m) e^{-i \lambda m}\right|^{-2} \\
& =\prod_{\nu \in \mathcal{M}}\left|\left(e^{-i \nu}-e^{i \lambda}\right)\left(e^{i \nu}-e^{i \lambda}\right)\right|^{-2 \widetilde{D}_{\nu}}
\end{aligned}
$$

coefficients $G_{k^{*}}^{+}(m), G_{k^{*}}^{-}(m)$ are defined by (9), (10).

The spectral density $f(\lambda)$ and the structural function $D_{\bar{s}}^{(R)}(m, \overline{1}, \overline{1})$ of a stationary GM increment sequence $\chi_{\overline{1}, \bar{s}}^{(R)}(\vec{\xi}(m))$ exhibit the following behavior for the constant matrices $C$ and $K$ :

- $\left|\beta^{(R)}(i \lambda)\right|^{-2}\left|\chi_{1}^{(R)}\left(e^{-i \lambda}\right)\right|^{2} f(\lambda) \sim C|\nu-\lambda|^{-2 \widetilde{D}_{\nu}}$ when $\lambda \rightarrow \nu, \nu \in \mathcal{M}$ (for properties of eigenvalues of generalized fractional process, we refer to Palma and Bondon [42])

- $D_{\bar{s}}^{(R)}(m, \overline{1}, \overline{1}) \sim K \sum_{\nu \in \mathcal{M}: \widetilde{D}_{\nu}>0}|m|^{2 \widetilde{D}_{\nu}-1} \cos (m \nu)$, as $m \rightarrow \infty$, see Giraitis and Leipus [12].

Example 2

1. For the increment operator $(1-B)^{R_{0}+D_{0}}\left(1-B^{2}\right)^{R_{1}+D_{1}}, \mathcal{M}_{0}=\{0\}, \mathcal{M}_{1}=\{0, \pi\}, \mathcal{M}=\{0, \pi\}$, the Gegenbauer representation is $(1-B)^{D_{0}+D_{1}}(1+B)^{D_{1}}$. The stationarity conditions are as follows: $|D|=\mid D_{0}+$ $D_{1}|<1 / 2,| D_{\pi}|=| D_{1} \mid<1 / 2$.

2. For the increment operator $\left(1-B^{2}\right)^{R_{1}+D_{1}}\left(1-B^{3}\right)^{R_{2}+D_{2}}, \quad \mathcal{M}_{0}=\{0, \pi\}, \quad \mathcal{M}_{1}=\{0,2 \pi / 3\}, \quad \mathcal{M}=$ $\{0,2 \pi / 3, \pi\}$, the Gegenbauer representation is $(1-B)^{D_{1}+D_{2}}\left(1-2 \cos (2 \pi / 3) B+B^{2}\right)^{D_{2}}(1+B)^{D_{1}}$. The stationarity conditions are as follows: $|D|=\left|D_{1}+D_{2}\right|<1 / 2,\left|D_{2 \pi / 3}\right|=\left|D_{2}\right|<1 / 2,\left|D_{\pi}\right|=\left|D_{1}\right|<1 / 2$.

3. For the increment operator $\left(1-B^{2}\right)^{R_{1}+D_{1}}\left(1-B^{4}\right)^{R_{2}+D_{2}}, \mathcal{M}_{0}=\{0, \pi\}, \quad \mathcal{M}_{1}=\{0, \pi / 2, \pi\}, \quad \mathcal{M}=$ $\{0, \pi / 2, \pi\}$, the Gegenbauer representation is $(1-B)^{D_{1}+D_{2}}\left(1+B^{2}\right)^{D_{2}}(1+B)^{D_{1}+D_{2}}$. The stationarity conditions are as follows: $|D|=\left|D_{\pi}\right|=\left|D_{1}+D_{2}\right|<1 / 2,\left|D_{\pi / 2}\right|=\left|D_{2}\right|<1 / 2$.

\section{Hilbert space projection method of filtering}

\subsection{Filtering of vector-valued stochastic sequence with stationary GM increments}

Consider a vector-valued stochastic sequence $\vec{\xi}(m)$ with stationary GM increments constructed from transformation (4) and a vector-valued stationary stochastic sequence $\vec{\eta}(m)$ uncorrelated with the sequence $\vec{\xi}(m)$. Let the stationary GM increment sequence $\chi_{\bar{\mu}, \bar{s}}^{(d)}(\vec{\xi}(m))=\left\{\chi_{\bar{\mu}, \bar{s}}^{(d)}\left(\xi_{p}(m)\right)\right\}_{p=1}^{T}$ and the stationary sequence $\vec{\eta}(m)$ have absolutely continuous spectral functions $F(\lambda)$ and $G(\lambda)$ with the spectral densities $f(\lambda)=\left\{f_{i j}(\lambda)\right\}_{i, j=1}^{T}$ and $g(\lambda)=\left\{g_{i j}(\lambda)\right\}_{i, j=1}^{T}$ respectively. Without loss of generality assume that $\mathrm{E} \chi_{\bar{\mu}, \bar{s}}^{(d)}(\vec{\xi}(m))=0, \mathrm{E} \vec{\eta}(m)=0$ and $\bar{\mu}>\overline{0}$.

Filtering problem. Consider the problem of mean square optimal linear estimation of the functional

$$
A \vec{\xi}=\sum_{k=0}^{\infty}(\vec{a}(k))^{\top} \vec{\xi}(-k),
$$


which depends on unobserved values of a stochastic sequence $\vec{\xi}(k)=\left\{\xi_{p}(k)\right\}_{p=1}^{T}$ with stationary GM increments. Estimates are based on observations of the sequence $\vec{\zeta}(k)=\vec{\xi}(k)+\vec{\eta}(k)$ at points $k=0,-1,-2, \ldots$

We will suppose that the following conditions are satisfied:

- conditions on coefficients $\vec{a}(k)=\left\{a_{p}(k)\right\}_{p=1}^{T}, k \geq 0$

$$
\sum_{k=0}^{\infty}\|\vec{a}(k)\|<\infty, \quad \sum_{k=0}^{\infty}(k+1)\|\vec{a}(k)\|^{2}<\infty,
$$

- the minimality condition on the spectral densities $f(\lambda)$ and $g(\lambda)$

$$
\int_{-\pi}^{\pi} \operatorname{Tr}\left[\frac{\left|\beta^{(d)}(i \lambda)\right|^{2}}{\left|\chi_{\bar{\mu}}^{(d)}\left(e^{-i \lambda}\right)\right|^{2}}\left(f(\lambda)+\left|\beta^{(d)}(i \lambda)\right|^{2} g(\lambda)\right)^{-1}\right] d \lambda<\infty .
$$

The second condition (13) is the necessary and sufficient one under which the mean square error of the optimal estimate of functional $A \vec{\xi}$ is not equal to 0 .

We apply the Hilbert space estimation technique proposed by Kolmogorov [26] which can be described as a 3-stage procedure: (i) define a target element (to be estimated) of the space $H=L_{2}(\Omega, \mathcal{F}, \mathrm{P})$ of random variables $\gamma$ which have zero mean values and finite variances, $\mathrm{E} \gamma=0, \mathrm{E}|\gamma|^{2}<\infty$, endowed with the inner product $\left\langle\gamma_{1}, \gamma_{2}\right\rangle=\mathrm{E} \gamma_{1} \overline{\gamma_{2}}$, (ii) define a subspace of $H=L_{2}(\Omega, \mathcal{F}, \mathrm{P})$ generated by observations, (iii) find an estimate of the target element as an orthogonal projection on the defined subspace.

Stage (i). We first represent the functional $A \vec{\xi}$ as $A \vec{\xi}=A \vec{\zeta}-A \vec{\eta}$, where

$$
A \vec{\zeta}=\sum_{k=0}^{\infty}(\vec{a}(k))^{\top} \vec{\zeta}(-k), \quad A \vec{\eta}=\sum_{k=0}^{\infty}(\vec{a}(k))^{\top} \vec{\eta}(-k) .
$$

Note, that under conditions (12), the functional $A \vec{\eta}$ belongs to the space $H$. To find the optimal estimate of the functional $A \vec{\xi}$, it is sufficient to find the estimate of the functional $A \vec{\eta}$

$$
\widehat{A} \vec{\xi}=A \vec{\zeta}-\widehat{A} \vec{\eta},
$$

since the functional $A \vec{\zeta}$ depends on values of the stochastic sequence $\zeta(k)$ which is observed. The mean square errors of the optimal estimates $\widehat{A} \vec{\xi}$ and $\widehat{A} \vec{\eta}$ are connected by the relation

$$
\begin{aligned}
\Delta(f, g ; \widehat{A} \vec{\xi}) & =\mathrm{E}|A \vec{\xi}-\widehat{A} \vec{\xi}|^{2}=\mathrm{E}|A \vec{\zeta}-A \vec{\eta}-A \vec{\zeta}+\widehat{A} \vec{\eta}|^{2} \\
& =\mathrm{E}|A \vec{\eta}-\widehat{A} \vec{\eta}|^{2}=\Delta(f, g ; \widehat{A} \vec{\eta}) .
\end{aligned}
$$

Relation (14) implies that any linear estimate $\widehat{A} \vec{\xi}$ of the functional $A \vec{\xi}$ allows the representation

$$
\widehat{A} \vec{\xi}=\sum_{k=0}^{\infty}(\vec{a}(k))^{\top}(\vec{\xi}(-k)+\vec{\eta}(-k))-\int_{-\pi}^{\pi}\left(\vec{h}_{\bar{\mu}}(\lambda)\right)^{\top} d Z_{\xi^{(n)}+\eta^{(n)}}(\lambda),
$$

where $\vec{h}_{\bar{\mu}}(\lambda)=\left\{h_{p}(\lambda)\right\}_{p=1}^{T}$ is the spectral characteristic of the estimate $\widehat{A} \vec{\xi}$.

Stage (ii). The set of the observed GM increments $\left\{\chi_{\bar{\mu}, \bar{s}}^{(d)}(\vec{\xi}(k))+\chi_{\bar{\mu}, \bar{s}}^{(d)}(\vec{\eta}(k)): k \leq 0\right\}, \vec{\mu}>\overrightarrow{0}$ generates a closed linear subspace $H^{0}\left(\xi^{(d)}+\eta^{(d)}\right)$ of the Hilbert space $H=L_{2}(\Omega, \mathfrak{F}, \mathrm{P})$. The functions

$$
e^{i \lambda k} \chi_{\bar{\mu}}^{(d)}\left(e^{-i \lambda}\right) \frac{1}{\beta^{(d)}(i \lambda)} \vec{\delta}_{l}, \quad \vec{\delta}_{l}=\left\{\delta_{l p}\right\}_{p=1}^{T}, l=1, \ldots, T, k \leq 0,
$$


generate a closed linear subspace $L_{2}^{0}(p)$ of the Hilbert space $L_{2}(p)$. The relation

$$
\chi_{\bar{\mu}, \bar{s}}^{(d)}(\vec{\xi}(k))+\chi_{\bar{\mu}, \bar{s}}^{(d)}(\vec{\eta}(k))=\int_{-\pi}^{\pi} e^{i \lambda k} \chi_{\bar{\mu}}^{(d)}\left(e^{-i \lambda}\right) \frac{1}{\beta^{(d)}(i \lambda)} d Z_{\xi^{(d)}+\eta^{(d)}}(\lambda)
$$

implies a map between the elements $e^{i \lambda k} \chi_{\bar{\mu}}^{(d)}\left(e^{-i \lambda}\right)\left(\beta^{(d)}(i \lambda)\right)^{-1} \vec{\delta}_{l}$ from the space $L_{2}^{0}(p)$ and th elements $\chi_{\bar{\mu}, \bar{s}}^{(d)}(\vec{\xi}(k))+\chi_{\bar{\mu}, \bar{s}}^{(d)}(\vec{\eta}(k))$ from the space $H^{0}\left(\xi^{(d)}+\eta^{(d)}\right)$ respectively.

Stage (iii). The estimate $\widehat{A} \vec{\eta}$ is found as an orthogonal projection of the functional $A \vec{\eta}$ from the space $H=$ $L_{2}(\Omega, \mathfrak{F}, \mathrm{P})$ on the subspace $H^{0}\left(\xi^{(d)}+\eta^{(d)}\right)$. This projection is characterized by the following conditions:

1) $\widehat{A} \vec{\eta} \in H^{0}\left(\xi^{(d)}+\eta^{(d)}\right)$;

2) $(A \vec{\eta}-\widehat{A} \vec{\eta}) \perp H^{0}\left(\xi^{(d)}+\eta^{(d)}\right)$.

Define the following matrix-valued Fourier coefficients:

$$
\begin{aligned}
S_{k, j}^{\bar{\mu}} & =\frac{1}{2 \pi} \int_{-\pi}^{\pi} e^{-i \lambda(k+j)} \frac{\left|\beta^{(d)}(i \lambda)\right|^{2}}{\left|\chi_{\bar{\mu}}^{(d)}\left(e^{-i \lambda}\right)\right|^{2}} g(\lambda) p^{-1}(\lambda) d \lambda, \quad k \geq 0, j \geq-n(\gamma), \\
P_{k, j}^{\bar{\mu}} & =\frac{1}{2 \pi} \int_{-\pi}^{\pi} e^{i \lambda(j-k)} \frac{\left|\beta^{(d)}(i \lambda)\right|^{2}}{\left|\chi_{\bar{\mu}}^{(d)}\left(e^{-i \lambda}\right)\right|^{2}} p^{-1}(\lambda) d \lambda, \quad k, j \geq 0, \\
Q_{k, j} & =\frac{1}{2 \pi} \int_{-\pi}^{\pi} e^{i \lambda(j-k)} f(\lambda) g(\lambda) p^{-1}(\lambda) d \lambda, \quad k, j \geq 0 .
\end{aligned}
$$

Define the vectors a $=\left((\vec{a}(0))^{\top},(\vec{a}(1))^{\top},(\vec{a}(2))^{\top}, \ldots\right)^{\top}$ and $\mathbf{a}_{\bar{\mu}}=\left(\left(\vec{a}_{\bar{\mu}}(0)\right)^{\top},\left(\vec{a}_{\bar{\mu}}(1)\right)^{\top},\left(\vec{a}_{\bar{\mu}}(2)\right)^{\top}, \ldots\right)^{\top}$, where the coefficients $\vec{a}_{\bar{\mu}}(k)=\vec{a}_{-\bar{\mu}}(k-n(\gamma)), k \geq 0$,

$$
\vec{a}_{-\bar{\mu}}(m)=\sum_{l=\max \{m, 0\}}^{m+n(\gamma)} e_{\gamma}(l-m) \vec{a}(l), \quad m \geq-n(\gamma) .
$$

Denote by $\mathbf{P}_{\bar{\mu}}, \mathbf{S}_{\bar{\mu}}$ and $\mathbf{Q}$ matrices with the matrix-values entries $\left(\mathbf{P}_{\bar{\mu}}\right)_{l, k}=P_{l, k}^{\bar{\mu}},\left(\mathbf{S}_{\bar{\mu}}\right)_{l, k}=S_{l+1, k-n(\gamma)}^{\bar{\mu}},(\mathbf{Q})_{l, k}=$ $Q_{l, k}, l, k \geq 0$.

Theorem 3

A solution $\widehat{A} \vec{\xi}$ to the filtering problem for the linear functional $A \vec{\xi}$ of the values of a vector-valued stochastic sequence $\vec{\xi}(m)$ with stationary GM increments under conditions (12) and (13) is calculated by formula (15). The spectral characteristic $\vec{h}_{\bar{\mu}}(\lambda)$ and the value of the mean square error $\Delta(f, g ; \widehat{A} \vec{\xi})$ are calculated by the formulas

$$
\left(\vec{h}_{\bar{\mu}}(\lambda)\right)^{\top}=\left[\chi_{\bar{\mu}}^{(d)}\left(e^{i \lambda}\right)\left(A\left(e^{-i \lambda}\right)\right)^{\top} g(\lambda)-\left(C_{\bar{\mu}}\left(e^{i \lambda}\right)\right)^{\top}\right] p^{-1}(\lambda) \frac{\overline{\beta^{(d)}(i \lambda)}}{\chi_{\bar{\mu}}^{(d)}\left(e^{i \lambda}\right)},
$$

where

$$
A\left(e^{-i \lambda}\right)=\sum_{k=0}^{\infty} \vec{a}(k) e^{-i \lambda k}, \quad C_{\bar{\mu}}\left(e^{i \lambda}\right)=\sum_{k=0}^{\infty}\left(\mathbf{P}_{\bar{\mu}}^{-1} \mathbf{S}_{\bar{\mu}} \mathbf{a}_{\bar{\mu}}\right)_{k} e^{i \lambda(k+1)}
$$


and

$$
\begin{aligned}
& \Delta(f, g ; \widehat{A} \vec{\xi})=\Delta(f, g ; \widehat{A} \vec{\eta})=\mathrm{E}|A \vec{\eta}-\widehat{A} \vec{\eta}|^{2} \\
& =\frac{1}{2 \pi} \int_{-\pi}^{\pi} \frac{1}{\left|\chi_{\bar{\mu}}^{(d)}\left(e^{-i \lambda}\right)\right|^{2}}\left(\chi_{\bar{\mu}}^{(d)}\left(e^{i \lambda}\right)\left(A\left(e^{-i \lambda}\right)\right)^{\top} f(\lambda)+\left|\beta^{(d)}(i \lambda)\right|^{2}\left(C_{\bar{\mu}}\left(e^{i \lambda}\right)\right)^{\top}\right) p^{-1}(\lambda) g(\lambda) p^{-1}(\lambda) \\
& \quad \times\left(\chi_{\bar{\mu}}^{(d)}\left(e^{-i \lambda}\right) f(\lambda) A\left(e^{i \lambda}\right)+\left|\beta^{(d)}(i \lambda)\right|^{2} C_{\bar{\mu}}\left(e^{-i \lambda}\right)\right) d \lambda \\
& +\frac{1}{2 \pi} \int_{-\pi}^{\pi} \frac{\left|\beta^{(d)}(i \lambda)\right|^{2}}{\left|\chi_{\bar{\mu}}^{(d)}\left(e^{-i \lambda}\right)\right|^{2}}\left(\chi_{\bar{\mu}}^{(d)}\left(e^{i \lambda}\right)\left(A\left(e^{-i \lambda}\right)\right)^{\top} g(\lambda)-\left(C_{\bar{\mu}}\left(e^{i \lambda}\right)\right)^{\top}\right) p^{-1}(\lambda) f(\lambda) p^{-1}(\lambda) \\
& \quad \times\left(\chi_{\bar{\mu}}^{(d)}\left(e^{-i \lambda}\right) g(\lambda) A\left(e^{i \lambda}\right)-C_{\bar{\mu}}\left(e^{-i \lambda}\right)\right) d \lambda \\
& =\left\langle\mathbf{S}_{\bar{\mu}} \mathbf{a}_{\bar{\mu}}, \mathbf{P}_{\bar{\mu}}^{-1} \mathbf{S}_{\bar{\mu}} \mathbf{a}_{\bar{\mu}}\right\rangle+\langle\mathbf{Q} \mathbf{a}, \mathbf{a}\rangle .
\end{aligned}
$$

Proof

See Appendix.

The filtering problem for the functional $A_{N} \vec{\xi}$ is solved directly by Theorem 3 by putting $\vec{a}(k)=\overrightarrow{0}$ for $k>N$. To solve the filtering problem for the $p$ th coordinate of the single vector $\vec{\xi}(-N)$, we put $\vec{a}(N)=\vec{\delta}_{p}, \vec{a}(k)=\overrightarrow{0}$ for $k \neq N$.

Define a matrix $\mathbf{S}_{\bar{\mu}, N}$ with the $T \times T$ matrix entries $\left(\mathbf{S}_{\bar{\mu}, N}\right)_{l, m}=S_{l+1, m-n(\gamma)}^{\bar{\mu}}$ for $l \geq 0,0 \leq m \leq N+n(\gamma)$ and $\left(\mathbf{S}_{\bar{\mu}, N}\right)_{l, m}=0$ for $l \geq 0, m>N+n(\gamma)$. Define another matrix $\mathbf{Q}_{N}$ with the $T \times T$ matrix entries $\left(\mathbf{Q}_{N}\right)_{l, k}=Q_{l, k}$, $0 \leq l, k \leq N$, and $\left(\mathbf{Q}_{N}\right)_{l, k}=0$ otherwise.

The following corollaries take place.

Corollary 1

A solution $\widehat{A}_{N} \vec{\xi}$ to the filtering problem for the linear functional $A_{N} \vec{\xi}$ of the values of a vector-valued stochastic sequence $\vec{\xi}(m)$ with stationary GM increments under condition (12) is calculated by the formula

$$
\widehat{A}_{N} \vec{\xi}=\sum_{k=0}^{N}(\vec{a}(k))^{\top}(\vec{\xi}(-k)+\vec{\eta}(-k))-\int_{-\pi}^{\pi}\left(h_{\bar{\mu}, N}(\lambda)\right)^{\top} d Z_{\xi^{(n)}+\eta^{(n)}}(\lambda) .
$$

The spectral characteristic $\vec{h}_{\bar{\mu}, N}(\lambda)$ of the optimal estimate $\widehat{A}_{N} \vec{\xi}$ is calculated by the formula

$$
\left(\vec{h}_{\bar{\mu}}(\lambda)\right)^{\top}=\left[\chi_{\bar{\mu}}^{(d)}\left(e^{i \lambda}\right)\left(A_{N}\left(e^{-i \lambda}\right)\right)^{\top} g(\lambda)-\left(C_{N}^{\bar{\mu}}\left(e^{i \lambda}\right)\right)^{\top}\right] p^{-1}(\lambda) \frac{\overline{\beta^{(d)}(i \lambda)}}{\chi_{\bar{\mu}}^{(d)}\left(e^{i \lambda}\right)},
$$

where

$$
A_{N}\left(e^{i \lambda}\right)=\sum_{k=0}^{N} \vec{a}(k) e^{-i \lambda k}, \quad C_{N}^{\bar{\mu}}\left(e^{i \lambda}\right)=\sum_{k=0}^{\infty}\left(\mathbf{P}_{\bar{\mu}}^{-1} \mathbf{S}_{\bar{\mu}, N} \mathbf{a}_{\bar{\mu}, N}\right)_{k} e^{i \lambda(k+1)},
$$

and

$$
\begin{aligned}
\overrightarrow{\mathbf{a}}_{\bar{\mu}, N} & =\left(\vec{a}_{\bar{\mu}, N}(0), \vec{a}_{\bar{\mu}, N}(1), \ldots, \vec{a}_{\bar{\mu}, N}(N+n(\gamma)), 0, \ldots\right)^{\top}, \\
\vec{a}_{\bar{\mu}, N}(k) & =\vec{a}_{-\bar{\mu}, N}(k-n(\gamma)), \quad 0 \leq k \leq N+n(\gamma), \\
\vec{a}_{-\bar{\mu}, N}(m) & =\sum_{l=\max \{m, 0\}}^{\min \{m+n(\gamma), N\}} e_{\gamma}(l-m) \vec{a}(l), \quad-n(\gamma) \leq m \leq N .
\end{aligned}
$$

The value of the mean square error $\Delta\left(f, g ; \widehat{A}_{N} \vec{\xi}\right)$ is calculated by the formula

$$
\begin{aligned}
\Delta\left(f, g ; \widehat{A}_{N} \vec{\xi}\right) & =\Delta\left(f, g ; \widehat{A} \vec{\eta}_{N}\right)=\mathrm{E}\left|A \vec{\eta}_{N}-\widehat{A} \vec{\eta}_{N}\right|^{2} \\
& =\left\langle\mathbf{S}_{\bar{\mu}, N} \mathbf{a}_{\bar{\mu}, N}, \mathbf{P}_{\bar{\mu}}^{-1} \mathbf{S}_{\bar{\mu}, N} \mathbf{a}_{\bar{\mu}, N}\right\rangle+\left\langle\mathbf{Q}_{N} \mathbf{a}_{N}, \mathbf{a}_{N}\right\rangle,
\end{aligned}
$$


where $\mathbf{a}_{N}=\left((\vec{a}(0))^{\top},(\vec{a}(1))^{\top},(\vec{a}(2))^{\top}, \ldots,(\vec{a}(N))^{\top}, 0, \ldots\right)^{\top}$.

Corollary 2

The optimal linear estimate $\widehat{\xi}_{p}(-N)$ of an unobserved value $\xi_{p}(-N), N \geq 0$, of the stochastic vector sequence $\vec{\xi}(m)$ with GM stationary increments based on observations of the sequence $\vec{\xi}(m)+\vec{\eta}(m)$ at points $m=$ $0,-1,-2, \ldots$, where the noise sequence $\vec{\eta}(m)$ is uncorrelated with $\vec{\xi}(m)$, is calculated by the formula

$$
\widehat{\xi}_{p}(-N)=\left(\xi_{p}(-N)+\eta_{p}(-N)\right)-\int_{-\pi}^{\pi}\left(\vec{h}_{\bar{\mu}, N, p}\right)^{\top}(\lambda) d Z_{\xi^{(n)}+\eta^{(n)}}(\lambda) .
$$

The spectral characteristic of the estimate $\widehat{\xi}_{p}(-N)$ is calculated by the formula

$$
\left(\vec{h}_{\bar{\mu}, N, p}(\lambda)\right)^{\top}=\left[e^{-i \lambda N} \chi_{\bar{\mu}}^{(d)}\left(e^{i \lambda}\right)\left(\vec{\delta}_{p}\right)^{\top} g(\lambda)-\left(C_{N, p}^{\bar{\mu}}\left(e^{i \lambda}\right)\right)^{\top}\right] p^{-1}(\lambda) \frac{\overline{\beta^{(d)}(i \lambda)}}{\chi_{\bar{\mu}}^{(d)}\left(e^{i \lambda}\right)},
$$

where

$$
C_{N, p}^{\bar{\mu}}\left(e^{i \lambda}\right)=\sum_{k=0}^{\infty}\left(\mathbf{P}_{\bar{\mu}}^{-1} \mathbf{S}_{\mu, N} \overrightarrow{\mathbf{a}}_{\bar{\mu}, N, p}\right)_{k} e^{i \lambda(k+1)}
$$

and

$$
\begin{aligned}
\overrightarrow{\mathbf{a}}_{\bar{\mu}, N, p} & =\left(0, \ldots, 0,\left(\vec{a}_{\bar{\mu}, N, p}(N)\right)^{\top},\left(\vec{a}_{\bar{\mu}, N, p}(N+1)\right)^{\top}, \ldots,\left(\vec{a}_{\bar{\mu}, N, p}(N+n(\gamma))\right)^{\top}, 0, \ldots\right)^{\top}, \\
\vec{a}_{\bar{\mu}, N, p}(k) & =\vec{a}_{-\bar{\mu}, N, p}(k-n(\gamma)), \quad N \leq k \leq N+n(\gamma), \\
\vec{a}_{-\bar{\mu}, N, p}(m) & =e_{\gamma}(N-m) \vec{\delta}_{p}, \quad N-n(\gamma) \leq m \leq N .
\end{aligned}
$$

The value of the mean square error of the estimate $\widehat{\xi}_{p}(-N)$ is calculated by the formula

$$
\begin{aligned}
\Delta\left(f, g ; \widehat{\xi}_{p}(-N)\right) & =\Delta\left(f, g ; \widehat{\eta}_{p}(-N)\right)=\mathrm{E}\left|\eta_{p}(-N)-\widehat{\eta}_{p}(-N)\right|^{2} \\
& =\left\langle\mathbf{S}_{\bar{\mu}, N} \mathbf{a}_{\bar{\mu}, N . p}, \mathbf{P}_{\bar{\mu}}^{-1} \mathbf{S}_{\bar{\mu}, N} \mathbf{a}_{\bar{\mu}, N, p}\right\rangle+\left\langle Q_{0,0} \vec{\delta}_{p}, \vec{\delta}_{p}\right\rangle .
\end{aligned}
$$

Remark 2

The filtering problem in the presence of fractional integration can be solved using Theorem 3 and Corollaries 1, 2 under conditions of Theorem 2 on the increment orders $d_{i}$.

\subsection{Filtering of stochastic sequences with periodically stationary GM increment}

Consider the filtering problem for the functionals

$$
A \xi=\sum_{k=0}^{\infty} a^{(\xi)}(k) \xi(-k), \quad A_{M} \xi=\sum_{k=0}^{N} a^{(\xi)}(k) \xi(-k)
$$

which depend on unobserved values of a stochastic sequence $\xi(m)$ with periodically stationary GM increments. Estimates are based on observations of the sequence $\xi(m)+\eta(m)$ at points $m=0,-1,-2, \ldots$, where the periodically stationary noise sequence $\eta(m)$ is uncorrelated with $\xi(m)$.

The functional $A \xi$ can be represented in the form

$$
\begin{aligned}
A \xi & =\sum_{k=0}^{\infty} a^{(\xi)}(k) \xi(-k)=\sum_{m=0}^{\infty} \sum_{p=1}^{T} a^{(\xi)}(m T+p-1) \xi(-m T-p+1) \\
& =\sum_{m=0}^{\infty} \sum_{p=1}^{T} a_{p}(m) \xi_{p}(-m)=\sum_{m=0}^{\infty}(\vec{a}(m))^{\top} \vec{\xi}(-m)=A \vec{\xi},
\end{aligned}
$$


where

$$
\vec{\xi}(m)=\left(\xi_{1}(m), \xi_{2}(m), \ldots, \xi_{T}(m)\right)^{\top}, \xi_{p}(m)=\xi(m T+p-1) ; p=1,2, \ldots, T
$$

and

$$
\vec{a}(m)=\left(a_{1}(m), a_{2}(m), \ldots, a_{T}(m)\right)^{\top}, a_{p}(m)=a^{(\xi)}(m T+p-1) ; p=1,2, \ldots, T .
$$

In the same way, the functional $A \eta$ is represented as

$$
A \eta=\sum_{k=0}^{\infty} a^{(\xi)}(k) \eta(-k)=\sum_{m=0}^{\infty}(\vec{a}(m))^{\top} \vec{\eta}(-m)=A \vec{\eta},
$$

where

$$
\vec{\eta}(m)=\left(\eta_{1}(m), \eta_{2}(m), \ldots, \eta_{T}(m)\right)^{\top}, \eta_{p}(m)=\eta(m T+p-1) ; p=1,2, \ldots, T .
$$

Making use of the introduced notations and statements of Theorem 3 we can claim that the following theorem holds true.

\section{Theorem 4}

Let a stochastic sequence $\xi(m)$ with periodically stationary GM increments and a stochastic periodically stationary sequence $\eta(m)$ generate by formulas (27) and (29) vector-valued stochastic sequences $\vec{\xi}(m)$ and $\vec{\eta}(m)$ with the spectral densities matrices $f(\lambda)=\left\{f_{i j}(\lambda)\right\}_{i, j=1}^{T}$ and $g(\lambda)=\left\{g_{i j}(\lambda)\right\}_{i, j=1}^{T}$. A solution $\widehat{A} \xi$ to the filtering problem for the functional $A \xi=A \vec{\xi}$ under conditions (12) and (13) is calculated by formula (15) for the coefficients $\vec{a}(m)$, $m \geq 0$, defined in (28). The spectral characteristic $\vec{h}_{\bar{\mu}}(\lambda)=\left\{h_{p}(\lambda)\right\}_{p=1}^{T}$ and the value of the mean square error $\Delta(f ; \widehat{A} \xi)$ of the estimate $\widehat{A} \xi$ are calculated by formulas (17) and (18) respectively.

The functional $A_{M} \xi$ can be represented in the form

$$
\begin{aligned}
A_{M} \xi & =\sum_{k=0}^{M} a^{(\xi)}(k) \zeta(-k)=\sum_{m=0}^{N} \sum_{p=1}^{T} a^{(\xi)}(m T+p-1) \xi(-m T-p+1) \\
& =\sum_{m=0}^{N} \sum_{p=1}^{T} a_{p}(m) \xi_{p}(-m)=\sum_{m=0}^{N}(\vec{a}(m))^{\top} \vec{\xi}(-m)=A_{N} \vec{\xi},
\end{aligned}
$$

where $N=\left[\frac{M}{T}\right]$, the sequence $\vec{\xi}(m)$ is determined by formula (27),

$$
\begin{aligned}
(\vec{a}(m))^{\top} & =\left(a_{1}(m), a_{2}(m), \ldots, a_{T}(m)\right)^{\top}, \\
a_{p}(m) & =a^{\zeta}(m T+p-1) ; 0 \leq m \leq N ; 1 \leq p \leq T ; m T+p-1 \leq M ; \\
a_{p}(N) & =0 ; \quad M+1 \leq N T+p-1 \leq(N+1) T-1 ; 1 \leq p \leq T .
\end{aligned}
$$

An estimate of a single unobserved value $\xi(-M), M \geq 0$ of a stochastic sequence $\xi(m)$ with periodically stationary GM increments is obtained by making use of the notations $\xi(-M)=\xi_{p}(-N)=\left(\vec{\delta}_{p}\right)^{\top} \vec{\xi}(N), N=\left[\frac{M}{T}\right]$, $p=M+1-N T$. We can conclude that the following corollaries hold true.

\section{Corollary 3}

Let a stochastic sequence $\xi(m)$ with periodically stationary GM increments and a stochastic periodically stationary sequence $\eta(m)$ generate by formulas (27) and (29) vector-valued stochastic sequences $\vec{\xi}(m)$ and $\vec{\eta}(m)$. A solution $\widehat{A}_{M} \xi$ to the filtering problem for the functional $A_{M} \xi=A_{N} \vec{\xi}$ under condition (13) is calculated by formula (19) for the coefficients $\vec{a}(m), 0 \leq m \leq N$, defined in (30). The spectral characteristic and the value of the mean square error of the estimate $\widehat{A}_{M} \xi$ are calculated by formulas (20) and (22) respectively.

\section{Corollary 4}

Let a stochastic sequence $\xi(m)$ with periodically stationary GM increments and a stochastic periodically stationary 
sequence $\eta(m)$ generate by formulas (27) and (29) vector-valued stochastic sequences $\vec{\xi}(m)$ and $\vec{\eta}(m)$. A solution $\widehat{\xi}(-M)$ to the filtering problem for an unobserved value $\xi(-M)=\xi_{p}(-N)=\left(\vec{\delta}_{p}\right)^{\top} \vec{\xi}(-N), N=\left[\frac{M}{T}\right]$, $p=M+1-N T$, under condition (13) is calculated by formula (23). The spectral characteristic and the value of the mean square error of the estimate $\widehat{\xi}(-M)$ are calculated by formulas (24) and (25) respectively.

\section{Minimax (robust) method of filtering}

Solutions of the problem of estimating the functionals $A \vec{\xi}$ and $A_{N} \vec{\xi}$ constructed from unobserved values of the stochastic sequence $\vec{\xi}(m)$ with stationary GM increments $\chi_{\bar{\mu}, \bar{s}}^{(d)}(\vec{\xi}(m))$ having the spectral density matrix $f(\lambda)$ based on its observations with stationary noise $\vec{\xi}(m)+\vec{\eta}(m)$ at points $m=0,-1,-2, \ldots$ are proposed in Theorem 3 and Corollary 1 in the case where the spectral density matrices $f(\lambda)$ and $g(\lambda)$ of the target sequence and the noise are exactly known.

In this section, we study the case where the complete information about the spectral density matrices is not available, while some sets of admissible spectral densities $\mathcal{D}=\mathcal{D}_{f} \times \mathcal{D}_{g}$ is known. The minimax approach of estimation of the functionals from unobserved values of stochastic sequences is considered, which consists in finding an estimate that minimizes the maximal values of the mean square errors for all spectral densities from a class $\mathcal{D}$ simultaneously. This method will be applied for the concrete classes of spectral densities.

The proceed with the stated problem, we recall the following definitions [35].

\section{Definition 4}

For a given class of spectral densities $\mathcal{D}=\mathcal{D}_{f} \times \mathcal{D}_{g}$, the spectral densities $f^{0}(\lambda) \in \mathcal{D}_{f}, g^{0}(\lambda) \in \mathcal{D}_{g}$ are called the least favourable densities in the class $\mathcal{D}$ for optimal linear filtering of the functional $A \xi$ if the following relation holds true

$$
\Delta\left(f^{0}, g^{0}\right)=\Delta\left(h\left(f^{0}, g^{0}\right) ; f^{0}, g^{0}\right)=\max _{(f, g) \in \mathcal{D}_{f} \times \mathcal{D}_{g}} \Delta(h(f, g) ; f, g) .
$$

\section{Definition 5}

For a given class of spectral densities $\mathcal{D}=\mathcal{D}_{f} \times \mathcal{D}_{g}$ the spectral characteristic $\vec{h}^{0}(\lambda)$ of the optimal estimate of the functional $A \xi$ is called minimax (robust) if the following relations hold true

$$
\begin{aligned}
\vec{h}^{0}(\lambda) \in H_{\mathcal{D}} & =\bigcap_{(f, g) \in \mathcal{D}_{f} \times \mathcal{D}_{g}} L_{2}^{0}(p), \\
\min _{\vec{h} \in H_{\mathcal{D}}} \max _{(f, g) \in \mathcal{D}_{f} \times \mathcal{D}_{g}} \Delta(\vec{h} ; f, g) & =\max _{(f, g) \in \mathcal{D}_{f} \times \mathcal{D}_{g}} \Delta\left(\vec{h}^{0} ; f, g\right) .
\end{aligned}
$$

Taking into account the introduced definitions and the relations derived in the previous sections we can verify that the following lemma holds true.

\section{Lemma 2}

The spectral densities $f^{0} \in \mathcal{D}_{f}, g^{0} \in \mathcal{D}_{g}$ which satisfy the minimality condition (13) are the least favourable spectral densities in the class $\mathcal{D}$ for the optimal linear filtering of the functional $A \xi$ if the matrices $\mathbf{P}_{\bar{\mu}}^{0}, \mathbf{S}_{\bar{\mu}}^{0}, \mathbf{Q}^{0}$ defined by the Fourier coefficients of the functions

$$
\left|\beta^{(d)}(i \lambda)\right|^{2}\left|\chi_{\bar{\mu}}^{(d)}\left(e^{-i \lambda}\right)\right|^{-2}\left(p^{0}(\lambda)\right)^{-1}, \quad\left|\beta^{(d)}(i \lambda)\right|^{2}\left|\chi_{\bar{\mu}}^{(d)}\left(e^{-i \lambda}\right)\right|^{-2} g^{0}(\lambda)\left(p^{0}(\lambda)\right)^{-1}, \quad f^{0}(\lambda) g^{0}(\lambda)\left(p^{0}(\lambda)\right)^{-1},
$$

where

$$
p^{0}(\lambda):=f^{0}(\lambda)+\left|\beta^{(d)}(i \lambda)\right|^{2} g^{0}(\lambda),
$$

determine a solution of the constrained optimization problem

$$
\max _{(f, g) \in \mathcal{D}_{f} \times \mathcal{D}_{g}}\left(\left\langle\mathbf{S}_{\bar{\mu}} \mathbf{a}_{\bar{\mu}}, \mathbf{P}_{\bar{\mu}}^{-1} \mathbf{S}_{\bar{\mu}} \mathbf{a}_{\bar{\mu}}\right\rangle+\langle\mathbf{Q} \mathbf{a}, \mathbf{a}\rangle\right)=\left\langle\mathbf{S}_{\bar{\mu}}^{0} \mathbf{a}_{\bar{\mu}},\left(\mathbf{P}_{\bar{\mu}}^{0}\right)^{-1} \mathbf{S}_{\bar{\mu}}^{0} \mathbf{a}_{\bar{\mu}}\right\rangle+\left\langle\mathbf{Q}^{0} \mathbf{a}, \mathbf{a}\right\rangle .
$$

The minimax spectral characteristic $h^{0}=h_{\bar{\mu}}\left(f^{0}, g^{0}\right)$ is calculated by formula (17) if $h_{\bar{\mu}}\left(f^{0}, g^{0}\right) \in H_{\mathcal{D}}$. 
The more detailed analysis of properties of the least favorable spectral densities and the minimax-robust spectral characteristics shows that the minimax spectral characteristic $h^{0}$ and the least favourable spectral densities $f^{0}$ and $g^{0}$ form a saddle point of the function $\Delta(h ; f, g)$ on the set $H_{\mathcal{D}} \times \mathcal{D}$. The saddle point inequalities

$$
\Delta\left(h ; f^{0}, g^{0}\right) \geq \Delta\left(h^{0} ; f^{0}, g^{0}\right) \geq \Delta\left(h^{0} ; f, g\right) \quad \forall(f, g) \in \mathcal{D}, \forall h \in H_{\mathcal{D}}
$$

hold true if $h^{0}=h_{\bar{\mu}}\left(f^{0}, g^{0}\right), h_{\bar{\mu}}\left(f^{0}, g^{0}\right) \in H_{\mathcal{D}}$ and $\left(f^{0}, g^{0}\right)$ is a solution of the constrained optimization problem

$$
\widetilde{\Delta}(f, g)=-\Delta\left(h_{\bar{\mu}}\left(f^{0}, g^{0}\right) ; f, g\right) \rightarrow \inf , \quad(f, g) \in \mathcal{D},
$$

where the functional $\Delta\left(h_{\bar{\mu}}\left(f^{0}, g^{0}\right) ; f, g\right)$ is calculated by the formula

$$
\begin{aligned}
& \Delta\left(h_{\bar{\mu}}\left(f^{0}, g^{0}\right) ; f, g\right) \\
= & \frac{1}{2 \pi} \int_{-\pi}^{\pi} \frac{\left|\beta^{(d)}(i \lambda)\right|^{2}}{\left|\chi_{\bar{\mu}}^{(d)}\left(e^{-i \lambda}\right)\right|^{2}}\left(\chi_{\bar{\mu}}^{(d)}\left(e^{i \lambda}\right)\left(A\left(e^{-i \lambda}\right)\right)^{\top} g^{0}(\lambda)-\left(C_{\bar{\mu}}^{0}\left(e^{i \lambda}\right)\right)^{\top}\right)\left(p^{0}(\lambda)\right)^{-1} f(\lambda)\left(p^{0}(\lambda)\right)^{-1} \\
& \times\left(\chi_{\bar{\mu}}^{(d)}\left(e^{-i \lambda}\right) g^{0}(\lambda) A\left(e^{i \lambda}\right)-C_{\bar{\mu}}^{0}\left(e^{-i \lambda}\right)\right) d \lambda \\
+ & \frac{1}{2 \pi} \int_{-\pi}^{\pi} \frac{1}{\left|\chi_{\bar{\mu}}^{(d)}\left(e^{-i \lambda}\right)\right|^{2}}\left(\chi_{\bar{\mu}}^{(d)}\left(e^{i \lambda}\right)\left(A\left(e^{-i \lambda}\right)\right)^{\top} f^{0}(\lambda)+\left|\beta^{(d)}(i \lambda)\right|^{2}\left(C_{\bar{\mu}}^{0}\left(e^{i \lambda}\right)\right)^{\top}\right)\left(p^{0}(\lambda)\right)^{-1} g(\lambda)\left(p^{0}(\lambda)\right)^{-1} \\
& \times\left(\chi_{\bar{\mu}}^{(d)}\left(e^{-i \lambda}\right) f^{0}(\lambda) A\left(e^{i \lambda}\right)+\left|\beta^{(d)}(i \lambda)\right|^{2} C_{\bar{\mu}}^{0}\left(e^{-i \lambda}\right)\right) d \lambda \\
= & \left\langle\mathbf{S}_{\bar{\mu}}^{0} \mathbf{a}_{\bar{\mu}},\left(\mathbf{P}_{\bar{\mu}}^{0}\right)^{-1} \mathbf{S}_{\bar{\mu}}^{0} \mathbf{a}_{\bar{\mu}}\right\rangle+\left\langle\mathbf{Q}^{0} \mathbf{a}, \mathbf{a}\right\rangle
\end{aligned}
$$

with

$$
C_{\bar{\mu}}^{0}\left(e^{i \lambda}\right)=\sum_{k=0}^{\infty}\left(\left(\mathbf{P}_{\bar{\mu}}^{0}\right)^{-1} \mathbf{S}_{\bar{\mu}}^{0} \mathbf{a}_{\bar{\mu}}\right)_{k} e^{i \lambda(k+1)}
$$

The constrained optimization problem (32) is equivalent to the unconstrained optimization problem

$$
\Delta_{\mathcal{D}}(f, g)=\widetilde{\Delta}(f, g)+\delta(f, g \mid \mathcal{D}) \rightarrow \inf
$$

where $\delta(f, g \mid \mathcal{D})$ is the indicator function of the set $\mathcal{D}$, namely $\delta(f, g \mid \mathcal{D})=0$ if $(f, g) \in \mathcal{D}$ and $\delta(f \mid \mathcal{D})=+\infty$ if $(f, g) \notin \mathcal{D}$. The condition $0 \in \partial \Delta_{\mathcal{D}}\left(f^{0}, g^{0}\right)$ characterizes a solution $\left(f^{0}, g^{0}\right)$ of the stated unconstrained optimization problem. This condition is the necessary and sufficient condition that the point $\left(f^{0}, g^{0}\right)$ belongs to the set of minimums of the convex functional $\Delta_{\mathcal{D}}(f, g)[36,46]$. Thus, it allows us to find the equalities for the least favourable spectral densities in some special classes of spectral densities $\mathcal{D}$.

The form of the functional $\Delta\left(h_{\bar{\mu}}\left(f^{0}, g^{0}\right) ; f, g\right)$ is suitable for application of the Lagrange method of indefinite multipliers to the constrained optimization problem (32). Thus, the complexity of the problem is reduced to finding the subdifferential of the indicator function of the set of admissible spectral densities. We illustrate the solving of the problem (34) for concrete sets admissible spectral densities in the following subsections. A semi-uncertain filtering problem, when the spectral density $f(\lambda)$ is known and the spectral density $g(\lambda)$ belongs to in class $\mathcal{D}_{g}$, is considered as well. 


\subsection{Least favorable spectral density in classes $\mathcal{D}_{0} \times \mathcal{D}_{V}^{U}$}

Consider the minimax filtering problem for the functional $A \vec{\xi}$ for sets of admissible spectral densities $\mathcal{D}_{0}^{k}$, $k=1,2,3,4$ of the sequence with GM increments $\vec{\xi}(m)$

$$
\begin{aligned}
& \mathcal{D}_{0}^{1}=\left\{f(\lambda) \mid \frac{1}{2 \pi} \int_{-\pi}^{\pi} \frac{\left|\chi_{\bar{\mu}}^{(d)}\left(e^{-i \lambda}\right)\right|^{2}}{\left|\beta^{(d)}(i \lambda)\right|^{2}} f(\lambda) d \lambda=P\right\}, \\
& \mathcal{D}_{0}^{2}=\left\{f(\lambda) \mid \frac{1}{2 \pi} \int_{-\pi}^{\pi} \frac{\left|\chi_{\bar{\mu}}^{(d)}\left(e^{-i \lambda}\right)\right|^{2}}{\left|\beta^{(d)}(i \lambda)\right|^{2}} \operatorname{Tr}[f(\lambda)] d \lambda=p\right\}, \\
& \mathcal{D}_{0}^{3}=\left\{f(\lambda) \mid \frac{1}{2 \pi} \int_{-\pi}^{\pi} \frac{\left|\chi_{\bar{\mu}}^{(d)}\left(e^{-i \lambda}\right)\right|^{2}}{\left|\beta^{(d)}(i \lambda)\right|^{2}} f_{k k}(\lambda) d \lambda=p_{k}, k=\overline{1, T}\right\}, \\
& \mathcal{D}_{0}^{4}=\left\{f(\lambda) \mid \frac{1}{2 \pi} \int_{-\pi}^{\pi} \frac{\left|\chi_{\bar{\mu}}^{(d)}\left(e^{-i \lambda}\right)\right|^{2}}{\left|\beta^{(d)}(i \lambda)\right|^{2}}\left\langle B_{1}, f(\lambda)\right\rangle d \lambda=p\right\},
\end{aligned}
$$

where $p, p_{k}, k=\overline{1, T}$ are given numbers, $P, B_{1}$ are given positive-definite Hermitian matrices, and sets of admissible spectral densities $\mathcal{D}_{V}^{U}, k=1,2,3,4$ for the stationary noise sequence $\vec{\eta}(m)$

$$
\begin{aligned}
& \mathcal{D}_{V}^{U^{1}}=\left\{g(\lambda) \mid V(\lambda) \leq g(\lambda) \leq U(\lambda), \frac{1}{2 \pi} \int_{-\pi}^{\pi} g(\lambda) d \lambda=Q\right\}, \\
& \mathcal{D}_{V}^{U^{2}}=\left\{g(\lambda) \mid \operatorname{Tr}[V(\lambda)] \leq \operatorname{Tr}[g(\lambda)] \leq \operatorname{Tr}[U(\lambda)], \frac{1}{2 \pi} \int_{-\pi}^{\pi} \operatorname{Tr}[g(\lambda)] d \lambda=q\right\}, \\
& \mathcal{D}_{V}^{U^{3}}=\left\{g(\lambda) \mid v_{k k}(\lambda) \leq g_{k k}(\lambda) \leq u_{k k}(\lambda), \frac{1}{2 \pi} \int_{-\pi}^{\pi} g_{k k}(\lambda) d \lambda=q_{k}, k=\overline{1, T}\right\}, \\
& \mathcal{D}_{V}^{U^{4}}=\left\{g(\lambda) \mid\left\langle B_{2}, V(\lambda)\right\rangle \leq\left\langle B_{2}, g(\lambda)\right\rangle \leq\left\langle B_{2}, U(\lambda)\right\rangle, \frac{1}{2 \pi} \int_{-\pi}^{\pi}\left\langle B_{2}, g(\lambda)\right\rangle d \lambda=q\right\},
\end{aligned}
$$

where the spectral densities $V(\lambda), U(\lambda)$ are known and fixed, $q, q_{k}, k=\overline{1, T}$ are given numbers, $Q, B_{2}$ are given positive definite Hermitian matrices.

From the condition $0 \in \partial \Delta_{\mathcal{D}}\left(f^{0}, g^{0}\right)$ we find the following equations which determine the least favourable spectral densities for these given sets of admissible spectral densities.

Define

$$
\begin{aligned}
C_{\bar{\mu}}^{f 0}\left(e^{i \lambda}\right) & :=\chi_{\bar{\mu}}^{(d)}\left(e^{i \lambda}\right)\left(A\left(e^{-i \lambda}\right)\right)^{\top} g^{0}(\lambda)-\left(C_{\bar{\mu}}^{0}\left(e^{i \lambda}\right)\right)^{\top}, \\
C_{\bar{\mu}}^{g 0}\left(e^{i \lambda}\right) & :=\frac{\left|\chi_{\bar{\mu}}^{(d)}\left(e^{-i \lambda}\right)\right|^{2}}{\left|\beta^{(d)}(i \lambda)\right|^{2}}\left(A\left(e^{-i \lambda}\right)\right)^{\top} f^{0}(\lambda)+\chi_{\bar{\mu}}^{(d)}\left(e^{-i \lambda}\right)\left(C_{\bar{\mu}}^{0}\left(e^{i \lambda}\right)\right)^{\top} .
\end{aligned}
$$

For the first pair of the sets of admissible spectral densities $\mathcal{D}_{f 0}^{1} \times \mathcal{D}_{V}^{U^{1}}$ we have equations

$$
\begin{aligned}
C_{\bar{\mu}}^{f 0}\left(e^{i \lambda}\right) C_{\bar{\mu}}^{f 0}\left(e^{i \lambda}\right)^{*} & =\left(\frac{\left|\chi_{\bar{\mu}}^{(d)}\left(e^{-i \lambda}\right)\right|^{2}}{\left|\beta^{(d)}(i \lambda)\right|^{2}} p^{0}(\lambda)\right) \vec{\alpha} \cdot \vec{\alpha}^{*}\left(\frac{\left|\chi_{\bar{\mu}}^{(d)}\left(e^{-i \lambda}\right)\right|^{2}}{\left|\beta^{(d)}(i \lambda)\right|^{2}} p^{0}(\lambda)\right) \\
C_{\bar{\mu}}^{g 0}\left(e^{i \lambda}\right) C_{\bar{\mu}}^{g 0}\left(e^{i \lambda}\right)^{*} & =\left(\frac{\left|\chi_{\bar{\mu}}^{(d)}\left(e^{-i \lambda}\right)\right|^{2}}{\left|\beta^{(d)}(i \lambda)\right|^{2}} p^{0}(\lambda)\right)\left(\vec{\beta} \cdot \vec{\beta}^{*}+\Gamma_{1}(\lambda)+\Gamma_{2}(\lambda)\right)\left(\frac{\left|\chi_{\bar{\mu}}^{(d)}\left(e^{-i \lambda}\right)\right|^{2}}{\left|\beta^{(d)}(i \lambda)\right|^{2}} p^{0}(\lambda)\right),
\end{aligned}
$$

where $\vec{\alpha}, \vec{\beta}$ are vectors of Lagrange multipliers, $\Gamma_{1}(\lambda) \leq 0$ and $\Gamma_{1}(\lambda)=0$ if $g^{0}(\lambda)>V(\lambda), \Gamma_{2}(\lambda) \geq 0$ and $\Gamma_{2}(\lambda)=0$ if $g^{0}(\lambda)<U(\lambda)$. 
For the second pair of the sets of admissible spectral densities $\mathcal{D}_{f 0}^{2} \times \mathcal{D}_{V}^{U^{2}}$ we have equation

$$
\begin{aligned}
& C_{\bar{\mu}}^{f 0}\left(e^{i \lambda}\right) C_{\bar{\mu}}^{f 0}\left(e^{i \lambda}\right)^{*}=\alpha^{2}\left(\frac{\left|\chi_{\bar{\mu}}^{(d)}\left(e^{-i \lambda}\right)\right|^{2}}{\left|\beta^{(d)}(i \lambda)\right|^{2}} p^{0}(\lambda)\right)^{2}, \\
& C_{\bar{\mu}}^{g 0}\left(e^{i \lambda}\right) C_{\bar{\mu}}^{g 0}\left(e^{i \lambda}\right)^{*}=\left(\beta^{2}+\gamma_{1}(\lambda)+\gamma_{2}(\lambda)\right)\left(\frac{\left|\chi_{\bar{\mu}}^{(d)}\left(e^{-i \lambda}\right)\right|^{2}}{\left|\beta^{(d)}(i \lambda)\right|^{2}} p^{0}(\lambda)\right)^{2},
\end{aligned}
$$

where $\alpha^{2}, \beta^{2}$ are Lagrange multipliers, $\gamma_{1}(\lambda) \leq 0$ and $\gamma_{1}(\lambda)=0$ if $\operatorname{Tr}\left[g^{0}(\lambda)\right]>\operatorname{Tr}[V(\lambda)], \gamma_{2}(\lambda) \geq 0$ and $\gamma_{2}(\lambda)=$ 0 if $\operatorname{Tr}\left[g^{0}(\lambda)\right]<\operatorname{Tr}[U(\lambda)]$.

For the third pair of the sets of admissible spectral densities $\mathcal{D}_{f 0}^{3} \times \mathcal{D}_{V}^{U^{3}}$ we have equation

$$
\begin{aligned}
& C_{\bar{\mu}}^{f 0}\left(e^{i \lambda}\right) C_{\bar{\mu}}^{f 0}\left(e^{i \lambda}\right)^{*}=\left(\frac{\left|\chi_{\bar{\mu}}^{(d)}\left(e^{-i \lambda}\right)\right|^{2}}{\left|\beta^{(d)}(i \lambda)\right|^{2}} p^{0}(\lambda)\right)\left\{\alpha_{k}^{2} \delta_{k l}\right\}_{k, l=1}^{T}\left(\frac{\left|\chi_{\bar{\mu}}^{(d)}\left(e^{-i \lambda}\right)\right|^{2}}{\left|\beta^{(d)}(i \lambda)\right|^{2}} p^{0}(\lambda)\right) \\
& C_{\bar{\mu}}^{g 0}\left(e^{i \lambda}\right) C_{\bar{\mu}}^{g 0}\left(e^{i \lambda}\right)^{*}=\left(\frac{\left|\chi_{\bar{\mu}}^{(d)}\left(e^{-i \lambda}\right)\right|^{2}}{\left|\beta^{(d)}(i \lambda)\right|^{2}} p^{0}(\lambda)\right)\left\{\left(\beta_{k}^{2}+\gamma_{1 k}(\lambda)+\gamma_{2 k}(\lambda)\right) \delta_{k l}\right\}_{k, l=1}^{T}\left(\frac{\left|\chi_{\bar{\mu}}^{(d)}\left(e^{-i \lambda}\right)\right|^{2}}{\left|\beta^{(d)}(i \lambda)\right|^{2}} p^{0}(\lambda)\right)
\end{aligned}
$$

where $\alpha_{k}^{2}, \beta_{k}^{2}$ are Lagrange multipliers, $\delta_{k l}$ are Kronecker symbols, $\gamma_{1 k}(\lambda) \leq 0$ and $\gamma_{1 k}(\lambda)=0$ if $g_{k k}^{0}(\lambda)>v_{k k}(\lambda)$, $\gamma_{2 k}(\lambda) \geq 0$ and $\gamma_{2 k}(\lambda)=0$ if $g_{k k}^{0}(\lambda)<u_{k k}(\lambda)$.

For the fourth pair of the sets of admissible spectral densities $\mathcal{D}_{f 0}^{4} \times \mathcal{D}_{V}^{U^{4}}$ we have equation

$$
\begin{aligned}
C_{\bar{\mu}}^{f 0}\left(e^{i \lambda}\right) C_{\bar{\mu}}^{f 0}\left(e^{i \lambda}\right)^{*} & =\alpha^{2}\left(\frac{\left|\chi_{\bar{\mu}}^{(d)}\left(e^{-i \lambda}\right)\right|^{2}}{\left|\beta^{(d)}(i \lambda)\right|^{2}} p^{0}(\lambda)\right) B_{1}^{\top}\left(\frac{\left|\chi_{\bar{\mu}}^{(d)}\left(e^{-i \lambda}\right)\right|^{2}}{\left|\beta^{(d)}(i \lambda)\right|^{2}} p^{0}(\lambda)\right) \\
C_{\bar{\mu}}^{g 0}\left(e^{i \lambda}\right) C_{\bar{\mu}}^{g 0}\left(e^{i \lambda}\right)^{*} & =\left(\beta^{2}+\gamma_{1}^{\prime}(\lambda)+\gamma_{2}^{\prime}(\lambda)\right)\left(\frac{\left|\chi_{\bar{\mu}}^{(d)}\left(e^{-i \lambda}\right)\right|^{2}}{\left|\beta^{(d)}(i \lambda)\right|^{2}} p^{0}(\lambda)\right) B_{2}^{\top}\left(\frac{\left|\chi_{\bar{\mu}}^{(d)}\left(e^{-i \lambda}\right)\right|^{2}}{\left|\beta^{(d)}(i \lambda)\right|^{2}} p^{0}(\lambda)\right)
\end{aligned}
$$

where $\alpha^{2}, \beta^{2}$ are Lagrange multipliers, $\gamma_{1}^{\prime}(\lambda) \leq 0$ and $\gamma_{1}^{\prime}(\lambda)=0$ if $\left\langle B_{2}, g^{0}(\lambda)\right\rangle>\left\langle B_{2}, V(\lambda)\right\rangle, \gamma_{2}^{\prime}(\lambda) \geq 0$ and $\gamma_{2}^{\prime}(\lambda)=0$ if $\left\langle B_{2}, g^{0}(\lambda)\right\rangle<\left\langle B_{2}, U(\lambda)\right\rangle$.

The following theorem holds true.

\section{Theorem 5}

The least favorable spectral densities $f^{0}(\lambda)$ and $g^{0}(\lambda)$ in the classes $\mathcal{D}_{0}^{k} \times \mathcal{D}_{V}^{U^{k}}, k=1,2,3,4$, for the optimal linear filtering of the functional $A \vec{\xi}$ are determined by pairs of equations (35)-(36), (37)-(38), (39)-(40), (41)(42), the minimality condition (13), the constrained optimization problem (31) and restrictions on densities from the corresponding classes $\mathcal{D}_{0}^{k} \times \mathcal{D}_{V}^{U^{k}}, k=1,2,3,4$. The minimax-robust spectral characteristic $h_{\bar{\mu}}\left(f^{0}, g^{0}\right)$ of the optimal estimate of the functional $A \vec{\xi}$ is determined by the formula (17).

\subsection{Semi-uncertain filtering problem in classes $\mathcal{D}_{\varepsilon}$ of least favorable noise spectral density}

Consider a semi-uncertain filtering problem for the functional $A \vec{\xi}$, where the spectral density $f(\lambda)$ of the sequence with GM increments $\vec{\xi}(m)$ is known and the spectral density $g(\lambda)$ of the stationary noise sequence $\vec{\eta}(m)$ belongs to the sets of admissible spectral densities $\mathcal{D}_{\varepsilon}^{k}, k=1,2,3,4$

$$
\begin{aligned}
& \mathcal{D}_{\varepsilon}^{1}=\left\{g(\lambda) \mid \operatorname{Tr}[g(\lambda)]=(1-\varepsilon) \operatorname{Tr}\left[g_{1}(\lambda)\right]+\varepsilon \operatorname{Tr}[W(\lambda)], \frac{1}{2 \pi} \int_{-\pi}^{\pi} \operatorname{Tr}[g(\lambda)] d \lambda=q\right\} ; \\
& \mathcal{D}_{\varepsilon}^{2}=\left\{g(\lambda) \mid g_{k k}(\lambda)=(1-\varepsilon) g_{k k}^{1}(\lambda)+\varepsilon w_{k k}(\lambda), \frac{1}{2 \pi} \int_{-\pi}^{\pi} g_{k k}(\lambda) d \lambda=q_{k}, k=\overline{1, T}\right\} ;
\end{aligned}
$$




$$
\begin{aligned}
& \mathcal{D}_{\varepsilon}^{3}=\left\{g(\lambda) \mid\left\langle B_{1}, g(\lambda)\right\rangle=(1-\varepsilon)\left\langle B_{2}, g_{1}(\lambda)\right\rangle+\varepsilon\left\langle B_{2}, W(\lambda)\right\rangle, \frac{1}{2 \pi} \int_{-\pi}^{\pi}\left\langle B_{2}, g(\lambda)\right\rangle d \lambda=q\right\} ; \\
& \mathcal{D}_{\varepsilon}^{4}=\left\{g(\lambda) \mid g(\lambda)=(1-\varepsilon) g_{1}(\lambda)+\varepsilon W(\lambda), \frac{1}{2 \pi} \int_{-\pi}^{\pi} g(\lambda) d \lambda=Q\right\},
\end{aligned}
$$

where $g_{1}(\lambda)$ is a fixed spectral density, $W(\lambda)$ is an unknown spectral density, $q, q_{k}, k=\overline{1, T}$, are given numbers, $Q, B_{2}$ are given positive-definite Hermitian matrices.

The condition $0 \in \partial \Delta_{\mathcal{D}}\left(f, g^{0}\right)$ implies the equations which determine the least favourable spectral densities of the noise sequence $\vec{\eta}(m)$.

Define

$$
C_{\bar{\mu}}^{g, s e m i}\left(e^{i \lambda}\right):=\frac{\left|\chi_{\bar{\mu}}^{(d)}\left(e^{-i \lambda}\right)\right|^{2}}{\left|\beta^{(d)}(i \lambda)\right|^{2}}\left(A\left(e^{-i \lambda}\right)\right)^{\top} f(\lambda)+\chi_{\bar{\mu}}^{(d)}\left(e^{-i \lambda}\right)\left(C_{\bar{\mu}}^{s e m i}\left(e^{i \lambda}\right)\right)^{\top},
$$

where $C_{\bar{\mu}}^{s e m i}\left(e^{i \lambda}\right)$ is determined by the matrices $\mathbf{P}_{\bar{\mu}}^{s e m i}, \mathbf{S}_{\bar{\mu}}^{s e m i}$ defined by the Fourier coefficients of the functions

$$
\begin{gathered}
\left|\beta^{(d)}(i \lambda)\right|^{2}\left|\chi_{\bar{\mu}}^{(d)}\left(e^{-i \lambda}\right)\right|^{-2}\left(f(\lambda)+\left|\beta^{(d)}(i \lambda)\right|^{2} g^{0}(\lambda)\right)^{-1}, \\
\left|\beta^{(d)}(i \lambda)\right|^{2}\left|\chi_{\bar{\mu}}^{(d)}\left(e^{-i \lambda}\right)\right|^{-2} g^{0}(\lambda)\left(f(\lambda)+\left|\beta^{(d)}(i \lambda)\right|^{2} g^{0}(\lambda)\right)^{-1} .
\end{gathered}
$$

For the sets of admissible spectral densities $\mathcal{D}_{\varepsilon}^{k}, k=1,2,3,4$, we have equations, respectively,

$$
C_{\bar{\mu}}^{g, s e m i}\left(e^{i \lambda}\right) C_{\bar{\mu}}^{g, s e m i}\left(e^{i \lambda}\right)^{*}=\left(\beta^{2}+\gamma_{2}(\lambda)\right)\left(\frac{\left|\chi_{\bar{\mu}}^{(d)}\left(e^{-i \lambda}\right)\right|^{2}}{\left|\beta^{(d)}(i \lambda)\right|^{2}}\left(f(\lambda)+\left|\beta^{(d)}(i \lambda)\right|^{2} g^{0}(\lambda)\right)\right)^{2}
$$

where $\beta^{2}$ is a Lagrange multiplier, $\gamma_{2}(\lambda) \leq 0$ and $\gamma_{2}(\lambda)=0$ if $\operatorname{Tr}\left[g^{0}(\lambda)\right]>(1-\varepsilon) \operatorname{Tr}\left[g_{1}(\lambda)\right]$,

$$
\begin{aligned}
C_{\bar{\mu}}^{g, s e m i}\left(e^{i \lambda}\right) C_{\bar{\mu}}^{g, s e m i}\left(e^{i \lambda}\right)^{*}=\frac{\left|\chi_{\bar{\mu}}^{(d)}\left(e^{-i \lambda}\right)\right|^{2}}{\left|\beta^{(d)}(i \lambda)\right|^{2}}\left(f(\lambda)+\left|\beta^{(d)}(i \lambda)\right|^{2} g^{0}(\lambda)\right) \\
\quad \times\left\{\left(\beta_{k}^{2}+\gamma_{k}^{2}(\lambda)\right) \delta_{k l}\right\}_{k, l=1}^{T} \frac{\left|\chi_{\bar{\mu}}^{(d)}\left(e^{-i \lambda}\right)\right|^{2}}{\left|\beta^{(d)}(i \lambda)\right|^{2}}\left(f(\lambda)+\left|\beta^{(d)}(i \lambda)\right|^{2} g^{0}(\lambda)\right),
\end{aligned}
$$

where $\beta_{k}^{2}$ are Lagrange multipliers, $\gamma_{k}^{2}(\lambda) \leq 0$ and $\gamma_{k}^{2}(\lambda)=0$ if $g_{k k}^{0}(\lambda)>(1-\varepsilon) g_{k k}^{1}(\lambda)$,

$$
\begin{aligned}
C_{\bar{\mu}}^{g, s e m i}\left(e^{i \lambda}\right) C_{\bar{\mu}}^{g, s e m i}\left(e^{i \lambda}\right)^{*}=\left(\beta^{2}+\gamma_{2}^{\prime}(\lambda)\right) \frac{\left|\chi_{\bar{\mu}}^{(d)}\left(e^{-i \lambda}\right)\right|^{2}}{\left|\beta^{(d)}(i \lambda)\right|^{2}} & \left(f(\lambda)+\left|\beta^{(d)}(i \lambda)\right|^{2} g^{0}(\lambda)\right) \\
& \times B_{2}^{\top} \frac{\left|\chi_{\bar{\mu}}^{(d)}\left(e^{-i \lambda}\right)\right|^{2}}{\left|\beta^{(d)}(i \lambda)\right|^{2}}\left(f(\lambda)+\left|\beta^{(d)}(i \lambda)\right|^{2} g^{0}(\lambda)\right),
\end{aligned}
$$

where $\beta^{2}$ is a Lagrange multiplier, $\gamma_{2}^{\prime}(\lambda) \leq 0$ and $\gamma_{2}^{\prime}(\lambda)=0$ if $\left\langle B_{2}, g^{0}(\lambda)\right\rangle>(1-\varepsilon)\left\langle B_{2}, g_{1}(\lambda)\right\rangle$,

$$
\begin{aligned}
& C_{\bar{\mu}}^{g, s e m i}\left(e^{i \lambda}\right) C_{\bar{\mu}}^{g, s e m i}\left(e^{i \lambda}\right)^{*}=\frac{\left|\chi_{\bar{\mu}}^{(d)}\left(e^{-i \lambda}\right)\right|^{2}}{\left|\beta^{(d)}(i \lambda)\right|^{2}}\left(f(\lambda)+\left|\beta^{(d)}(i \lambda)\right|^{2} g^{0}(\lambda)\right) \\
& \times\left(\vec{\beta} \cdot \vec{\beta}^{*}+\Gamma(\lambda)\right) \frac{\left|\chi_{\bar{\mu}}^{(d)}\left(e^{-i \lambda}\right)\right|^{2}}{\left|\beta^{(d)}(i \lambda)\right|^{2}}\left(f(\lambda)+\left|\beta^{(d)}(i \lambda)\right|^{2} g^{0}(\lambda)\right),
\end{aligned}
$$

where $\vec{\beta}$ is a vector of Lagrange multipliers, $\Gamma(\lambda) \leq 0$ and $\Gamma(\lambda)=0$ if $g^{0}(\lambda)>(1-\varepsilon) g_{1}(\lambda)$.

The following theorem holds true. 


\section{Theorem 6}

The least favorable spectral density $g^{0}(\lambda)$ in the classes $\mathcal{D}_{\varepsilon}^{k}, k=1,2,3,4$, for the optimal linear filtering of the functional $A \vec{\xi}$, where the spectral density $f(\lambda)$ of the sequence with GM increments $\vec{\xi}(m)$ is known, is determined by equations (43), (44), (45), (46), the minimality condition (13), the constrained optimization problem (31) for the fixed $f(\lambda)$ and the restrictions on density from the corresponding classes $\mathcal{D}_{\varepsilon}^{k}, k=1,2,3,4$. The minimax-robust spectral characteristic $h_{\bar{\mu}}\left(f, g^{0}\right)$ of the optimal estimate of the functional $A \vec{\xi}$ is determined by the formula (17).

\section{Conclusions}

In this article, we present methods of solution of the filtering problem for stochastic sequences with periodically stationary long memory multiple seasonal increments, or sequences with periodically stationary general multiplicative (GM) increments, introduced in the article by Luz and Moklyachuk [33]. These non-stationary stochastic sequences combine periodic structure of covariation functions of sequences as well as multiple seasonal factors, including the integrating one. A short review of the spectral theory of vector-valued generalized multiple increment sequences is presented. We describe methods of solution of the filtering problem in the case where the spectral densities of the sequence $\xi(m)$ and a noise sequence $\eta(m)$ are exactly known. Estimates are obtained by applying the Hilbert space projection technique to the vector sequence $\vec{\xi}(m)+\vec{\eta}(m)$ with stationary GM increments under the stationary noise sequence $\vec{\eta}(m)$ uncorrelated with $\vec{\xi}(m)$. The case of non-stationary fractional integration is discussed as well. The minimax-robust approach to filtering problem is applied in the case of spectral uncertainty where densities of sequences are not exactly known while, instead, sets of admissible spectral densities are specified. We propose a representation of the mean square error in the form of a linear functional in $L_{1}$ with respect to spectral densities, which allows us to solve the corresponding constrained optimization problem and describe the minimax (robust) estimates of the functionals. Relations are described which determine the least favourable spectral densities and the minimax spectral characteristics of the optimal estimates of linear functionals for a collection of specific classes of admissible spectral densities. These sets are generalizations of the sets of admissible spectral densities described in a survey article by Kassam and Poor [25] for stationary stochastic processes.

\section{Appendix}

\section{Proof of Theorem 3}

The condition $(A \vec{\eta}-\widehat{A} \vec{\eta}) \perp H^{0}\left(\xi^{(d)}+\eta^{(d)}\right)$ implies the relation which holds true for all $k \leq 0$ :

$$
\begin{aligned}
& \mathrm{E}(A \vec{\eta}-\widehat{A} \vec{\eta})^{\top}\left(\overline{\chi_{\bar{\mu}, \bar{s}}^{(d)}(\vec{\xi}(m))+\chi_{\bar{\mu}, \bar{s}}^{(d)}(\vec{\eta}(m))}\right) \\
= & \frac{1}{2 \pi} \int_{-\pi}^{\pi}\left(A\left(e^{-i \lambda}\right)-\beta^{(d)}(i \lambda) \vec{h}_{\bar{\mu}}(\lambda)\right)^{\top} g(\lambda) e^{-i \lambda k} \chi_{\bar{\mu}}^{(d)}\left(e^{i \lambda}\right) d \lambda \\
& -\frac{1}{2 \pi} \int_{-\pi}^{\pi}\left(\vec{h}_{\bar{\mu}}(\lambda)\right)^{\top} f(\lambda) e^{-i \lambda k} \chi_{\bar{\mu}}^{(d)}\left(e^{i \lambda}\right) \frac{1}{\overline{\beta^{(d)}(i \lambda)}} d \lambda=0 .
\end{aligned}
$$

Thus, for all $k \leq 0$, the function $\vec{h}_{\bar{\mu}}(\lambda)$ satisfies the relation

$$
\int_{-\pi}^{\pi}\left[\left(A\left(e^{-i \lambda}\right)\right)^{\top} g(\lambda) \overline{\beta^{(d)}(i \lambda)}-\left(\vec{h}_{\bar{\mu}}(\lambda)\right)^{\top} p(\lambda)\right] \frac{\chi_{\bar{\mu}}^{(d)}\left(e^{i \lambda}\right)}{\overline{\beta^{(d)}(i \lambda)}} e^{-i \lambda k} d \lambda=\overrightarrow{0} .
$$

The latter relation implies that the spectral characteristic $\vec{h}_{\bar{\mu}}(\lambda)$ allows a representation of the form (17) with

$$
C_{\bar{\mu}}\left(e^{i \lambda}\right)=\sum_{k=0}^{\infty} \vec{c}_{\bar{\mu}}(k) e^{i \lambda(k+1)},
$$


where $\vec{c}_{\bar{\mu}}(k)=\left\{c_{\bar{\mu} p}(k)\right\}_{p=1}^{T}, k \geq 0$, are unknown coefficients to be found.

The condition $\widehat{A} \vec{\eta} \in H^{0}\left(\xi^{(d)}+\eta^{(d)}\right)$ implies the following representation of the spectral characteristic $\vec{h}_{\bar{\mu}}(\lambda)$ :

$$
\vec{h}_{\bar{\mu}}(\lambda)=h(\lambda) \chi_{\bar{\mu}}^{(d)}\left(e^{-i \lambda}\right) \frac{1}{\beta^{(d)}(i \lambda)}, \quad h(\lambda)=\sum_{k=0}^{\infty} \vec{s}(k) e^{-i \lambda k},
$$

which leads to the relation holding true for all $l \geq 1$ :

$$
\int_{-\pi}^{\pi}\left[\chi_{\bar{\mu}}^{(d)}\left(e^{i \lambda}\right)\left(A\left(e^{-i \lambda}\right)\right)^{\top} g(\lambda)-\left(C_{\bar{\mu}}\left(e^{i \lambda}\right)\right)^{\top}\right] p^{-1}(\lambda) \frac{\left|\beta^{(d)}(i \lambda)\right|^{2}}{\left|\chi_{\bar{\mu}}^{(d)}\left(e^{-i \lambda}\right)\right|^{2}} e^{-i \lambda l} d \lambda=\overrightarrow{0} .
$$

In terms of the matrix-valued Fourier coefficients $S_{k, j}^{\bar{\mu}}$ for $k \geq 0, j \geq-n(\gamma), P_{k, j}^{\bar{\mu}}, Q_{k, j}$ for $k, j \geq 0$, relation (47) is presented as a system of linear equations

$$
\sum_{m=-n(\gamma)}^{\infty} S_{l+1, m}^{\bar{\mu}} \vec{a}_{-\bar{\mu}}(m)=\sum_{k=0}^{\infty} P_{l+1, k+1}^{\bar{\mu}} \vec{c}_{\bar{\mu}}(k), \quad l \geq 0,
$$

or in the matrix form as

$$
\mathbf{S}_{\bar{\mu}} \mathbf{a}_{\bar{\mu}}=\mathbf{P}_{\bar{\mu}} \mathbf{c}_{\bar{\mu}},
$$

where the vectors $\mathbf{c}_{\bar{\mu}}=\left(\left(\vec{c}_{\bar{\mu}}(0)\right)^{\top},\left(\vec{c}_{\bar{\mu}}(1)\right)^{\top},\left(\vec{c}_{\mu}(2)\right)^{\top}, \ldots\right)^{\top}, \mathbf{a}_{\bar{\mu}}=\left(\left(\vec{a}_{\bar{\mu}}(0)\right)^{\top},\left(\vec{a}_{\bar{\mu}}(1)\right)^{\top},\left(\vec{a}_{\bar{\mu}}(2)\right)^{\top}, \ldots\right)^{\top}$, the coefficients $a_{\bar{\mu}}(k)=a_{-\mu}(k-n(\gamma)), k \geq 0$ and the linear operators $\mathbf{P}_{\bar{\mu}}, \mathbf{S}_{\bar{\mu}}$ are defined in Subsection 3.1. Therefore, the unknown coefficients $\vec{c}_{\bar{\mu}}(k), k \geq 0$, are calculated by the formula

$$
\vec{c}_{\bar{\mu}}(k)=\left(\mathbf{P}_{\bar{\mu}}^{-1} \mathbf{S}_{\bar{\mu}} \mathbf{a}_{\bar{\mu}}\right)_{k}, \quad k \geq 0,
$$

where $\left(\mathbf{P}_{\bar{\mu}}^{-1} \mathbf{S}_{\bar{\mu}} \mathbf{a}_{\bar{\mu}}\right)_{k}, k \geq 0$, is the $k$ th $T$-dimensional vector element of the vector $\mathbf{P}_{\bar{\mu}}^{-1} \mathbf{S}_{\bar{\mu}} \mathbf{a}_{\bar{\mu}}$.

The derived expressions justifies the formulas (17) and (18) for calculating the spectral characteristic $\vec{h}_{\bar{\mu}}(\lambda)$ and the value of the mean square error $\Delta(f, g ; \widehat{A} \vec{\xi})$ of the estimate $\widehat{A} \vec{\xi}$ of the functional $A \vec{\xi}$.

\section{REFERENCES}

1. C. F. Ansley, and R. Kohn, Estimation, filtering and smoothing in state space models with incompletely specified initial conditions, The Annals of Statistics, vol. 13, pp. 1286-1316, 1985.

2. C. Baek, R. A. Davis, and V. Pipiras, Periodic dynamic factor models: estimation approaches and applications, Electronic Journal of Statistics, vol. 12, no. 2, pp. 4377-4411, 2018.

3. R. T. Baillie, C. Kongcharoen, and G. Kapetanios, Prediction from ARFIMA models: Comparisons between MLE and semiparametric estimation procedures, International Journal of Forecasting, vol. 28, pp. 46-53, 2012.

4. I.V. Basawa, R. Lund, and Q. Shao, First-order seasonal autoregressive processes with periodically varying parameters, Statistics and Probability Letters, vol. 67, no. 4, p. 299-306, 2004.

5. G. E. P. Box, G. M. Jenkins, G. C. Reinsel, and G.M. Ljung, Time series analysis. Forecasting and control. 5rd ed., John Wiley \& Sons, Hoboken, NJ, 2016.

6. P. Eiurridge, and K. F. Wallis, Seasonal adjustment and Kalman filtering: Extension to periodic variances, Journal of Forecasting, vol. 9, no. 2, p. 109-118, 1990.

7. I. I. Dubovets'ka, and M. P. Moklyachuk, Filtration of linear functionals of periodically correlated sequences, Theory of Probability and Mathematical Statistics, vol. 86, pp. 51-64, 2013.

8. G. Dudek, Forecasting time series with multiple seasonal cycles using neural networks with local learning, In: Rutkowski L., Korytkowski M., Scherer R., Tadeusiewicz R., Zadeh L.A., Zurada J.M. (eds) Artificial Intelligence and Soft Computing. ICAISC 2013. Lecture Notes in Computer Science, vol. 7894. Springer, Berlin, Heidelberg, pp. 52-63, 2013.

9. A. Dudek, H. Hurd, and W. Wojtowicz, PARMA methods based on Fourier representation of periodic coefficients, Wiley Interdisciplinary Reviews: Computational Statistics, vol. 8, no. 3, pp. 130-149, 2016.

10. J. Franke, Minimax-robust prediction of discrete time series, Z. Wahrscheinlichkeitstheor. Verw. Gebiete, vol. 68, no. 3, pp. 337-364, 1985.

11. I. I. Gikhman, and A. V. Skorokhod, The theory of stochastic processes. I., Springer, Berlin, 2004.

12. L. Giraitis, and R. Leipus, A generalized fractionally differencing approach in long-memory modeling, Lithuanian Mathematical Journal, vol. 35, pp. 53-65, 1995. 
13. E. G. Gladyshev, Periodically correlated random sequences, Sov. Math. Dokl. vol, 2, pp. 385-388, 1961.

14. P. G. Gould, A. B. Koehler, J. K. Ord, R. D. Snyder, R. J. Hyndman, and F. Vahid-Araghi, Forecasting time-series with multiple seasonal patterns, European Journal of Operational Research, vol. 191, pp. 207-222, 2008.

15. U. Grenander, A prediction problem in game theory, Arkiv för Matematik, vol. 3, pp. 371-379, 1957.

16. H. Gray, Q. Cheng and W. Woodward, On generalized fractional processes, Journal of Time Series Analysis, vol. 10, no. 3, pp. 233-257, 1989.

17. E. J. Hannan, Multiple time series. 2nd rev. ed., John Wiley \& Sons, New York, 2009.

18. U. Hassler, Time series analysis with long memory in view, Wiley, Hoboken, NJ, 2019.

19. U. Hassler, and M.O. Pohle, Forecasting under long memory and nonstationarity, arXiv:1910.08202, 2019.

20. Y. Hosoya, Robust linear extrapolations of second-order stationary processes, Annals of Probability, vol. 6, no. 4, pp. 574-584, 1978.

21. H. Hurd and V. Pipiras, Modeling periodic autoregressive time series with multiple periodic effects, In: Chaari F., Leskow J., Zimroz R., Wylomanska A., Dudek A. (eds) Cyclostationarity: Theory and Methods ?IV. CSTA 2017. Applied Condition Monitoring, vol 16. Springer, Cham, pp. 1-18, 2020.

22. S. Johansen, and M. O. Nielsen, The role of initial values in conditional sum-of-squaresestimation of nonstationary fractional time series models, Econometric Theory, vol. 32, no. 5, pp. 1095-1139, 2016.

23. K. Karhunen, Über lineare Methoden in der Wahrscheinlichkeitsrechnung, Annales Academiae Scientiarum Fennicae. Ser. A I, no. 37, 1947.

24. S. A. Kassam, Robust hypothesis testing and robust time series interpolation and regression, Journal of Time Series Analysis, vol. 3 , no. 3, pp. 185-194, 1982.

25. S. A. Kassam, and H. V. Poor, Robust techniques for signal processing: A survey, Proceedings of the IEEE, vol. 73, no. 3, pp. $1433-481,1985$.

26. A. N. Kolmogorov, Selected works by A. N. Kolmogorov. Vol. II: Probability theory and mathematical statistics. Ed. by A. N. Shiryayev. Mathematics and Its Applications. Soviet Series. 26. Dordrecht etc. Kluwer Academic Publishers, 1992.

27. P. S. Kozak, and M. P. Moklyachuk, Estimates of functionals constructed from random sequences with periodically stationary increments, Theory Probability and Mathematical Statistics, vol. 97, pp. 85-98, 2018.

28. Y. Liu, Yu. Xue and M. Taniguchi, Robust linear interpolation and extrapolation of stationary time series in Lp, Journal of Time Series Analysis, vol. 41, no. 2, pp. 229-248, 2020.

29. R. Lund, Choosing seasonal autocovariance structures: PARMA or SARMA, In: Bell WR, Holan SH, McElroy TS (eds) Economic time series: modelling and seasonality. Chapman and Hall, London, pp. 63-80, 2011.

30. M. Luz, and M. Moklyachuk, Minimax-robust filtering problem for stochastic sequence with stationary increments, Theory Probability and Mathematical Statistics, vol. 89, pp. 127-142, 2014.

31. M. Luz and M. Moklyachuk, Filtering problem for functionals of stationary sequences, Statistics, Optimization and Information Computing, vol. 4, no. 1, pp. 68?83, 2016.

32. M. Luz, and M. Moklyachuk, Estimation of stochastic processes with stationary increments and cointegrated sequences, London: ISTE; Hoboken, NJ: John Wiley \& Sons, 2019.

33. M. Luz and M. Moklyachuk, Minimax-robust forecasting of sequences with periodically stationary long memory multiple seasonal increments, Statistics, Optimization and Information Computing, vol. 8, no. 3, pp. 684-721, 2020.

34. O. Yu. Masyutka, M. P. Moklyachuk, and M. I. Sidei, Filtering of multidimensional stationary sequences with missing observations, Carpathian Mathematical Publications, vol. 11, no. 2, pp. 361-378, 2019.

35. M. P. Moklyachuk, Minimax filtration of linear transformations of stationary sequences, Ukrainian Mathematical Journal, vol. 43, pp. 75-81, 1991

36. M. P. Moklyachuk, Minimax-robust estimation problems for stationary stochastic sequences, Statistics, Optimization and Information Computing, vol. 3, no. 4, pp. 348-419, 2015.

37. M.P. Moklyachuk, and A. Yu. Masyutka, Robust filtering of stochastic processes Theory of Stochastic Processes, vol. 13, no. 1-2, pp. 166-181, 2007.

38. M. Moklyachuk, and M. Sidei, Filtering problem for stationary sequences with missing observations, Statistics, Optimization and Information Computing, vol. 4, no. 4, pp. 308-325, 2016.

39. M. Moklyachuk, M. Sidei, and O. Masyutka, Estimation of stochastic processes with missing observations, Mathematics Research Developments. Nova Science Publishers, New York, NY, 2019.

40. A. Napolitano, Cyclostationarity: New trends and applications, Signal Processing, vol. 120, pp. 385-408, 2016

41. D. Osborn, The implications of periodically varying coefficients for seasonal time-series processes, Journal of Econometrics, vol. 48 , no. 3, pp. 373-384, 1991.

42. W. Palma, and P. Bondon, On the eigenstructure of generalized fractional processes, Statistics and Probability Letters, vol. 65, pp. 93-101, 2003

43. S. Porter-Hudak, An application of the seasonal fractionally differenced model to the monetary aggegrates, Journal of the American Statistical Association, vol.85, no. 410, pp. 338-344, 1990.

44. V. A. Reisen, B. Zamprogno, W. Palma, and J. Arteche, A semiparametric approach to estimate two seasonal fractional parameters in the SARFIMA model, Mathematics and Computers in Simulation, vol. 98, pp. 1-17, 2014.

45. V. A. Reisen, E. Z. Monte, G. C. Franco, A. M. Sgrancio, F. A. F. Molinares, P. Bondond, F. A. Ziegelmann and B. Abraham, Robust estimation of fractional seasonal processes: Modeling and forecasting daily average SO2 concentrations, Mathematics and Computers in Simulation, vol. 146, pp. 27-43, 2018.

46. R. T. Rockafellar, Convex Analysis, Princeton Landmarks in Mathematics. Princeton University Press, Princeton, NJ, 1997.

47. C. C. Solci, V. A. Reisen, A. J. Q. Sarnaglia, and P. Bondon, Empirical study of robust estimation methods for PAR models with application to the air quality area, Communication in Statistics - Theory and Methods, vol. 48, no. 1, pp. 152-168, 2020.

48. H. Tsai, H. Rachinger, and E.M.H. Lin, Inference of seasonal long-memory time series with measurement error, Scandinavian Journal of Statistics, vol. 42, no. 1, pp. 137-154, 2015. 
49. S. K. Vastola, and H. V. Poor, Robust Wiener-Kolmogorov theory, IEEE Trans. Inform. Theory, vol. 30, no. 2, pp. 316-327, 1984.

50. A. M. Yaglom, Correlation theory of stationary and related random processes with stationary nth increments. American Mathematical Society Translations: Series 2, vol. 8, pp. 87-141, 1958.

51. A. M. Yaglom, Correlation theory of stationary and related random functions. Vol. 1: Basic results; Vol. 2: Supplementary notes and references, Springer Series in Statistics, Springer-Verlag, New York etc., 1987. 